Elsevier required licence: (c) <2019>. This manuscript version is made available under the CC-BY-NC-ND 4.0 license http://creativecommons.org/licenses/by-nc-nd/4.0/

The definitive publisher version is available online at https://doi.org/10.1016/j.jet.2019.04.009 


\title{
Efficient Investments in the Implementation Problem*
}

\author{
Kentaro Tomoeda ${ }^{\dagger}$
}

May 5, 2019

\begin{abstract}
This paper identifies a condition for an efficient social choice rule to be fully implementable when we take into account investment efficiency. To do so, we extend the standard implementation problem to include endogenous ex ante and ex post investments. In our problem, the social planner aims to achieve efficiency in every equilibrium of a dynamic game in which agents strategically make investments before and after playing the mechanism. Our main theorem shows that a novel condition commitment-proofness is sufficient and necessary for an efficient social choice rule to be implementable in subgame-perfect equilibria. The availability of ex post investments is crucial in our model: there is no social choice rule that is efficient and implementable in subgame-perfect equilibria without ex post investments. We also show that our positive result continues to hold in the incomplete information setting.
\end{abstract}

Keywords: investment efficiency, full implementation, mechanism design, ex ante investment, ex post investment

JEL Classification Numbers: D82, D44, D47, C78

${ }^{*}$ This was originally Chapter 1 of my $\mathrm{Ph} . \mathrm{D}$. thesis at Harvard University. I am very grateful to my advisors Eric Maskin, Scott Kominers and Edward Glaeser for their constant guidance. I would like to thank Masao Fukui, Chishio Furukawa, Ben Golub, Daisuke Hirata, Ryota Iijima, Sangram Kadam, Tetsuya Kaji, Michihiro Kandori, Yusuke Kasuya, Kohei Kawaguchi, Fuhito Kojima, Takashi Kunimoto, Jonathan Libgober, Simon Loertscher, Shintaro Miura, Xiaosheng Mu, Shunya Noda, Matthew Rabin, Ran Shorrer, Satoru Takahashi, Eduard Talamas, Tom Wilkening and Jun Xiao for their helpful discussions. Seminar participants at Kyoto University, Osaka University, Harvard University, Oxford University, University of Technology Sydney, GAMES 2016, University of New South Wales, Hitotsubashi University, Yokohama National University, National University of Singapore, University of Tokyo and University of Melbourne provided useful comments. I would also like to thank the editor, the associate editor and two anonymous referees for their helpful comments and suggestions.

$\dagger$ University of Technology Sydney, UTS Business School, PO Box 123, Broadway NSW 2007, Australia, Kentaro.Tomoeda@uts.edu.au. 


\section{Introduction}

The literature on implementation theory has identified which social choice rules can be fully implemented under various solution concepts and informational assumptions. Here, full implementation means that the set of all equilibrium outcomes of the mechanism coincides with the set of outcomes specified by the social choice rule. Although this is a strong requirement, the literature has shown rather permissive results: for example, under complete information and quasi-linear utility, any social choice rule is implementable in subgame-perfect equilibria (Moore and Repullo, 1988; Maskin and Tirole, 1999). ${ }^{1}$ In the implementation problem, however, the problem of investment incentives has not been fully examined. In many real-life applications such as auctions and the provision of public goods, there are opportunities for agents to invest in the outcomes of the mechanism (Tan, 1992; Bag, 1997; Arozamena and Cantillon, 2004). When agents strategically invest before participating in a mechanism, the positive results implied by implementation theory may be threatened. That is, although the mechanism implements efficient allocations at the market clearing stage, it may not necessarily induce efficient ex ante investments in equilibrium. In particular, even when there is an efficient investment equilibrium, we may not be able to rule out other inefficient equilibria, which is a concern of the full implementation problem.

The goal of this paper is to provide a condition for an "efficient" social choice rule to be implementable when we take into account both investment and allocative efficiency. ${ }^{2}$ To do so, we extend the standard implementation problem to include endogenous investments. First, we consider a rich set of types that are defined by the agents' costs of investment rather than their valuations of alternatives. Given realized cost types, agents endogenously form their valuations of the alternatives by investing before and after participating in the mechanism, which we call ex ante and ex post investments. ${ }^{3}$ Here, we explicitly model ex post investments because in many applications, agents make further investments after the market clearing stage to maximize the value of the outcome (McAfee and McMillan, 1986; Laffont and Tirole, 1986, 1987). A social choice rule $F$ is defined as a correspondence from the set of cost types to the set of alternatives, transfers and investments. This social choice rule, however, is not standard because investments are non-contractible and they are only

\footnotetext{
${ }^{1}$ There is a criticism that the Moore-Repullo mechanism is not robust to small perturbations of information (Aghion et al., 2012). In a more recent study, however, Chen et al. (2018) show that their mechanism can implement any social choice rule even with a certain class of information perturbations. Although these are important issues, we do not discuss robustness in this paper.

2 "Implementation" in this paper refers to full implementation.

${ }^{3}$ In Section 5, we consider the case where ex post investments are not possible.
} 
chosen by the agents as part of their strategies. We assume that the social planner can design a mechanism that specifies an alternative and a transfer vector, but that he cannot intervene in the structure of the investment opportunities. Therefore, the planner aims to achieve efficiency for every profile of cost types through a dynamic game in which agents strategically make ex ante and ex post investments in addition to playing the mechanism itself. In the main part of the paper, we assume that the agents have complete information. ${ }^{4}$

In this setting, our main theorem shows that the sufficient and necessary condition for an efficient social choice rule $F$ to be implementable in subgame-perfect equilibria is the commitment-proofness of the associated allocation and transfer rules $(\alpha, \tau)$ at the mechanism stage (Theorem 1). The associated $(\alpha, \tau)$ represents the choice of $F$ over contractible outcomes given the investments: these are the functions that specify the same alternative and transfer vector as $F$ for each profile of agents' valuation functions at the mechanism stage. Establishing a condition on $(\alpha, \tau)$ is useful for the social planner because $(\alpha, \tau)$ can be interpreted as a standard social choice function where investments are exogenously given.

To provide the intuition for Theorem 1, let us consider an example where a city decides on a public project to utilize a vacant lot. ${ }^{5}$ Suppose that there are several potential projects and that each citizen supports one of them. The goal of the city is to maximize social welfare from the lot taking into account the costs of the citizens' potential investments to utilize it. However, the investments and cost structures are neither observable to the city nor contractible. Suppose that some inefficient supporters have made a huge costly and irreversible investment in their favorite project prior to participating in the mechanism. Then, since the city does not know the cost of their ex ante investments and the cost is sunk, the city would simply choose their project as long as it is the most efficient one at the mechanism stage. However, there may be another project with more efficient supporters, which would require lower investment costs and hence achieve higher social welfare. This problem would not happen if none of the citizens had made any ex ante investments. Indeed, when the investment cost does not increase over time, the social efficiency can be achieved by making everyone invest only after the project is selected. ${ }^{6}$

How can the city prevent citizens from investing ex ante without directly intervening in their investment activities? Our solution is to design allocation and transfer rules $(\alpha, \tau)$ carefully so that they satisfy commitment-proofness. The commitment-proofness condition

\footnotetext{
${ }^{4}$ We also extend the model to the incomplete information setting. See Section 6.2.

${ }^{5}$ This example will be numerically elaborated in Section 3.

${ }^{6}$ In our model, we introduce time discounting $\delta \in(0,1)$ between two investment stages to rule out the possibility of any other investments being efficient.
} 
is interpreted in the following abstract way: suppose that (i) each citizen $i$ is assigned a "default" valuation $u_{i}$ for her favorite project, and (ii) $i$ could increase $u_{i}$ to $\tilde{u}_{i}$ ex ante through a certain commitment device that costs $\tilde{u}_{i}-u_{i}$. Even with this costly commitment device, a citizen may want to commit to a high valuation $\tilde{u}_{i}$ if her favorite project is selected only when she has $\tilde{u}_{i}$. The commitment-proofness of $(\alpha, \tau)$ requires that under $(\alpha, \tau)$, none of the citizens should have an incentive to change their valuation from any default $u_{i}$ to another $\tilde{u}_{i}$ through this commitment device. Going back to the investment problem in the example, for each citizen, the choice between no ex ante investment and making a costly investment corresponds to the choice between the default $u_{i}$ and another $\tilde{u}_{i}$ in the commitment-proofness condition. From this correspondence, none of the citizens has an incentive to invest ex ante under this condition, and the city succeeds in achieving efficiency. Commitment-proofness is a relatively weak requirement: it is implied by strategy-proofness, and moreover, we can find $(\alpha, \tau)$ that is commitment-proof, efficient and budget-balanced (Proposition 3).

The difficulty and novelty of our implementation problem stem from the combination of the following assumptions: (i) the investments are not contractible, (ii) the agents' cost types are not known to the planner, and (iii) the investments are irreversible. First, if the investments were contractible, they could just be part of the outcome of mechanisms and our problem reduces to the standard implementation problem. However, investment activities are usually difficult to describe; they are multidimensional and they involve the expenditure of time and effort as well as the expenditure of money (Hart, 1995). These non-contractible investments have also been a central concern in the literature on the hold-up problem (Klein et al., 1978; Williamson, 1979, 1983; Grossman and Hart, 1986; Hart and Moore, 1988, 1990). Second, if the planner knew the agents' cost types, he would be able to identify the first-best alternative in our model. Since the investments do not have externalities in our model, the efficient level of investment would be chosen by each agent if the planner just selects the first-best alternative. Finally, if the investments were reversible, ex ante investments would not affect the valuations of the alternatives at the mechanism stage. Therefore, the planner would not be bothered by ex ante investments, and the efficient choice of an alternative at the mechanism stage could simply achieve investment efficiency.

Our characterization result (Theorem 1) relies on the assumption that ex post investments are possible under the same cost functions. However, in many papers such as Rogerson (1992) and Hatfield et al. (2018), ex post investments are not explicitly considered. Hatfield et al. (2018) showed a result that is seemingly contradictory to our theorem: for efficient mechanisms, strategy-proofness is sufficient and necessary for the existence of an ex ante efficient investment equilibrium. The difference stems from the availability of ex post investments 
and more precisely, how it interacts with the implication of the rich cost types. In our model, since every agent takes into account an optimal ex post investment, there is a natural restriction to the set of possible valuations at the mechanism stage and their costs. On the other hand, in the necessity result of Hatfield et al. (2018), the richness of the cost types implies that any valuations could be associated with any costs. Therefore, the availability of ex post investments allows us to restrict the set of valuations and their costs at the mechanism stage in a natural way and obtain a more positive result. In Section 5, we examine our full implementation problem when ex post investments are not possible. We obtain an impossibility result in this setting: there does not exist a social choice rule that is efficient and implementable in subgame-perfect equilibria without ex post investments (Proposition $2){ }^{7}$

We also extend our main model to the incomplete information setting where agents are unsure about the cost types of other agents. In this environment, we show that an efficient social choice rule is implementable in PBE if its associated allocation and transfer rules are strategy-proof (Proposition 4).

\section{$1.1 \quad$ Related Literature}

Our research question mainly differs from those in the literature in the following two aspects. First, we extend the requirement of full implementation to the investment stage. The literature has mostly investigated the existence of an efficient investment equilibrium. Rogerson (1992) showed that when agents make pre-mechanism investments, there is a socially efficient investment equilibrium for any Bayesian incentive compatible and efficient mechanism. In the context of information acquisition (Milgrom, 1981; Obara, 2008), Bergemann and Välimäki (2002) provided a similar result: the VCG mechanism ensures ex ante efficiency under private values. Second, unlike most of the models in the incomplete-contracts literature (Grossman and Hart, 1986; Hart and Moore, 1988, 1990; Aghion et al., 1994), we assume that the cost types of agents are not known to the social planner. Hatfield et al. (2018) exploited the assumption of the rich cost types and showed the necessity of strategy-proofness for the existence of an efficient investment equilibrium.

There are several papers in the literature that analyzed ex ante investments under specific mechanisms such as the first-price and the second-price auctions (Tan, 1992; Piccione and

\footnotetext{
${ }^{7}$ On the other hand, if only ex post investments are possible, any efficient social choice rule is implementable in our model. This is because ex post investments are always chosen optimally in equilibrium, and extensive form mechanisms allow us to implement any alternatives and transfers by Moore and Repullo (1988).
} 
Tan, 1996; Stegeman, 1996; Bag, 1997; Arozamena and Cantillon, 2004; Loertscher and Riordan, 2019). A companion paper Tomoeda (2017) analyzed the implementability of efficient ex ante and ex post investments under the first-price auction. In contrast to these works, we take the approach of considering the entire space of social choice rules and identifying a condition for implementability.

There is also large body of the literature on investment incentives in bargaining and two-sided matching (Gul, 2001; Cole et al., 2001a,b; Felli and Roberts, 2002; de Meza and Lockwood, 2010; Mailath et al., 2013; Nöldeke and Samuelson, 2015). These papers usually do not model the possibility of ex post investments. This is because they focus on how agents bargain over the surplus of investments in the market clearing stage and their utility does not reflect their future investments. Hence, our main theorem may not be applied to their settings. That being said, our impossibility result without ex post investments applies to many of their models. Moreover, since investments are often allowed to have externalities in these models, it is even more difficult to eliminate inefficient investment equilibria due to coordination failure. For this reason, the question of full implementation is not typically asked in this strand of the literature.

The remainder of the paper is organized as follows. In Section 2, we introduce the formal model and provide the basic results. Section 3 discusses the intuition of our main results using a numerical example with two alternatives. In Section 4, commitment-proofness is introduced, and we present our main results. In Section 5, we provide an impossibility result when ex post investments are not possible. Section 6 contains two discussions about our main results: budget balance and incomplete information. Section 7 concludes. All proofs are in Appendix A.

\section{Model}

\section{$2.1 \quad$ Framework}

Consider a finite set $I$ of agents and a finite set $A$ of alternatives such that $|I| \geq 2$ and $|A| \geq 2$. The objective of the social planner is to maximize social welfare. As in the typical implementation problem, since the valuations of the alternatives are private information of the agents, the planner uses a mechanism to elicit the information. In our model, however, the valuations are endogenously determined: agents make investment decisions before and after participating in the mechanism to form their own valuations. We assume that these investments are observable among agents but are neither contractible nor observable to the 
social planner. This means that the planner can specify only an alternative and transfers in the mechanism but cannot directly specify investments. To maximize social welfare net of investment costs, the planner needs to take into account the equilibrium investment decisions by considering the investment game induced by the mechanism.

To describe each agent's investment decisions, we use a shortcut of modeling investment as an explicit choice of a valuation function $v_{i}: A \rightarrow \mathbb{R}^{8} V_{i} \subseteq \mathbb{R}^{A}$ is a compact set of all possible valuation functions for agent $i$, and we assume that there is $\kappa>0$ such that $[0, \kappa]^{A} \subseteq V_{i}$. Let $V \equiv \times_{i \in I} V_{i}$. The cost of investment is determined by cost function $c_{i}: \cup_{\theta_{i} \in \Theta_{i}}\left(V^{\theta_{i}} \times\left\{\theta_{i}\right\}\right) \rightarrow \mathbb{R}_{\geq 0}$, where $\Theta_{i}$ is the set of cost types of agent $i$ and $V^{\theta_{i}} \subseteq V_{i}$ is the set of possible valuation functions for type $\theta_{i}$. Let $\Theta \equiv \times_{i \in I} \Theta_{i}$ and $V^{\theta} \equiv \times_{i \in I} V^{\theta_{i}}$. Without loss of generality, the cost of investment is assumed to be non-negative, and for each $\theta_{i} \in \Theta_{i}$, there is $v_{i}^{0} \in V^{\theta_{i}}$ such that $c_{i}\left(v_{i}^{0}, \theta_{i}\right)=0$. The cost function for any agent $i$ is bounded; i.e., there exists $M \in \mathbb{R}_{\geq 0}$ such that $c_{i}\left(v_{i}, \theta_{i}\right) \leq M$ for any $\theta_{i} \in \Theta_{i}$ and $v_{i} \in V^{\theta_{i}}$. We impose the following two assumptions on the set of cost types $\Theta$ :

(Richness) For any compact set $W_{i} \subseteq V_{i}$ and any continuous function $r: W_{i} \rightarrow \mathbb{R}_{\geq 0}$, there exists $\theta_{i} \in \Theta_{i}$ such that $V^{\theta_{i}}=W_{i}$ and $c_{i}\left(\cdot, \theta_{i}\right)=r(\cdot)$.

(Compactness) For any $\theta_{i} \in \Theta_{i}$ and $v_{i} \in V^{\theta_{i}}, \bar{V}_{i}\left(v_{i}, \theta_{i}\right) \equiv\left\{\bar{v}_{i} \in V^{\theta_{i}} \mid c_{i}\left(\bar{v}_{i}, \theta_{i}\right) \geq c_{i}\left(v_{i}, \theta_{i}\right)\right\}$ is a compact set.

The richness condition is a key assumption used to show the necessity of commitmentproofness in Theorem 1 and the impossibility result in Proposition 2. The compactness condition ensures that we can always find an optimal choice of ex post investment.

We consider two timings of investment: before and after participating in the mechanism. Each investment stage is modeled as a simultaneous move game by all agents. ${ }^{9}$ Assume that the investment is irreversible: if agent $i$ with cost type $\theta_{i}$ chooses $v_{i}$ first, she can choose only a valuation function from the set $\bar{V}_{i}\left(v_{i}, \theta_{i}\right)=\left\{\bar{v}_{i} \in V^{\theta_{i}} \mid c_{i}\left(\bar{v}_{i}, \theta_{i}\right) \geq c_{i}\left(v_{i}, \theta_{i}\right)\right\}$ in the ex post investment stage. The timeline of the game is:

0. Agents observe their cost types $\theta \in \Theta$. The social planner announces a mechanism.

1. Each agent $i$ with cost type $\theta_{i}$ simultaneously chooses a valuation function $v_{i} \in V^{\theta_{i}}$.

2. Agents play the mechanism.

${ }^{8}$ This means that the investment does not have an externality effect on the other agents. See Matsushima and Noda (2016) for the case where investments have arbitrary externality effects on other agents' valuations.

${ }^{9}$ Our main results do not heavily rely on the simultaneity of the investments. Indeed, the main result (Theorem 1) continues to hold even when the agents make investments in an arbitrary order. 
3. After the mechanism is run, each agent $i$ with cost type $\theta_{i}$ can again choose a valuation function from $\bar{V}_{i}\left(v_{i}, \theta_{i}\right)$.

We call this the entire game induced by the mechanism. We also call the first stage of the game the ex ante investment stage, the second stage of the game the mechanism stage, and the third stage of the game the ex post investment stage.

The utility function of each agent is defined in the following way. Let $\delta \in(0,1)$ be a discount factor that discounts the utility realized in the second and third stages. ${ }^{10,11}$ For an alternative $a \in A$, a transfer vector $t \equiv\left(t_{i}\right)_{i \in I} \in \mathbb{R}^{I}$ and investments $\left(v_{i}, \bar{v}_{i}\right)$ where $v_{i}$ is the ex ante valuation and $\bar{v}_{i}$ is the ex post valuation, the ex ante utility of $i$ with cost type $\theta_{i}$ is defined by

$$
-c_{i}\left(v_{i}, \theta_{i}\right)+\delta\left[\bar{v}_{i}(a)-t_{i}-\left(c_{i}\left(\bar{v}_{i}, \theta_{i}\right)-c_{i}\left(v_{i}, \theta_{i}\right)\right)\right] .
$$

In the first stage, $i$ incurs the cost $c_{i}\left(v_{i}, \theta_{i}\right)$ of ex ante investment. In the second stage, the outcome $(a, t)$ of the mechanism is evaluated by the final valuation function $\bar{v}_{i}$. In the last stage, $i$ incurs the additional cost $c_{i}\left(\bar{v}_{i}, \theta_{i}\right)-c_{i}\left(v_{i}, \theta_{i}\right) \geq 0$ for revising the valuation function. ${ }^{12}$ Throughout the paper, we consider this quasi-linear utility function.

In the main part of the paper, we assume that the agents have complete information regarding the cost types. However, the social planner does not know their realized cost types. He only knows the environment, i.e., the set $I$ of agents, the set $A$ of alternatives, the set $V$ of possible valuation functions, the cost functions $c$ and the set $\Theta$ of possible cost types. Thus, the goal of the social planner is to implement efficient investments and allocations for all possible cost types $\theta \in \Theta$. We also assume that the chosen valuation functions are observable among the agents, but neither contractible nor observable to the planner. In Section 6.2, we also extend the model to the incomplete information setting.

In this model, a social choice rule $F: \Theta \rightarrow 2^{A \times \mathbb{R}^{I} \times V^{2}} \backslash\{\emptyset\}$ is defined as a non-empty valued correspondence from the set of cost types to the set of alternatives, transfer vectors and investments. Note that this social choice rule $F$ is not standard because the investments in $V^{2}$ are not contractible. Therefore, investments in $V^{2}$ are not achieved as an outcome of a mechanism but as strategies in the entire game induced by a mechanism. However, we still incorporate investments into an outcome of a social choice rule because our goal is to

\footnotetext{
${ }^{10}$ When $\delta=1$, the sufficiency of commitment-proofness does not hold in Theorem 1. See Observation 1.

${ }^{11}$ There is no time discounting between the mechanism stage and the ex post investment stage. However, this is without loss of generality because the set of cost types is rich.

${ }^{12}$ Here, we assume that the same cost function is used for both investment stages. Some of the main results, however, still hold when the cost functions differ over time. For example, the sufficiency part of Theorem 1 holds as long as the ex post cost function is weakly lower than the ex ante cost function.
} 
achieve the efficiency of both allocation and investment choices. The efficiency of a social choice rule $F$ requires that any $(a,(v, \bar{v}))$ in $F(\theta)$ should maximize the sum of the agents' valuations for an alternative net of the investment costs.

Definition 1. A social choice rule $F: \Theta \rightarrow 2^{A \times \mathbb{R}^{I} \times V^{2}} \backslash\{\emptyset\}$ is efficient if for any profile of cost types $\theta \in \Theta$,

$$
\begin{aligned}
& \left\{\left(a^{*},\left(v^{*}, \bar{v}^{*}\right)\right) \in A \times V^{2} \mid\left(a^{*}, t^{*},\left(v^{*}, \bar{v}^{*}\right)\right) \in F(\theta) \text { for some } t^{*} \in \mathbb{R}^{I}\right\} \\
& =\underset{(a,(v, \bar{v})) \in A \times\left\{(p, q) \in\left(V^{\theta}\right)^{2} \mid q \in \bar{V}(p, \theta)\right\}}{\arg \max } \sum_{i \in I}\left\{-c_{i}\left(v_{i}, \theta_{i}\right)+\delta\left[\bar{v}_{i}(a)-\left(c_{i}\left(\bar{v}_{i}, \theta_{i}\right)-c_{i}\left(v_{i}, \theta_{i}\right)\right)\right]\right\}
\end{aligned}
$$

where $\bar{V}(v, \theta) \equiv \times_{i \in I} \bar{V}_{i}\left(v_{i}, \theta_{i}\right)$.

Note that efficiency is defined in terms of the surplus generated through the entire game and that the transfers are not taken into account. ${ }^{13}$ One of the important implications of efficiency is that because $\delta$ is strictly less than one, the efficient choice of ex ante investment should always be $v^{*}$ with the lowest cost; i.e., for any $\theta \in \Theta$ and $\left(a^{*}, t^{*},\left(v^{*}, \bar{v}^{*}\right)\right) \in F(\theta)$, we have $v^{*} \in Z^{\theta} \equiv \times_{i \in I} Z^{\theta_{i}}$, where $Z^{\theta_{i}} \equiv\left\{v_{i} \in V^{\theta_{i}} \mid c_{i}\left(v_{i}, \theta_{i}\right)=0\right\}$.

To implement a social choice rule $F$, the social planner designs a mechanism. In our setting, we assume that the social planner can design a mechanism in the mechanism stage but cannot design the game of two investment stages. Therefore, given a mechanism, agents always face the entire game induced by the mechanism.

Since the focus of our paper is the implementability of efficient investments, we allow for flexibility in the form of the mechanisms. We consider general extensive form mechanisms because with them, any social choice of allocations can be implemented under complete information and quasi-linear utilities (Moore and Repullo, 1988). An extensive form mechanism $m$ is defined as a tuple $m \equiv(N,>, D, d, w)$, where $N$ is a set of nodes, $>$ is a partial ordering on $N$ that represents precedence, $D$ is a set of possible decisions, $d$ is a one-to-one function from $N \backslash\{n \in N \mid n$ has no predecessor $\}$ into $D$ that labels each non-initial node with the last decision taken to reach it, and $w \equiv\left(w^{\alpha}, w^{\tau}\right)$ is a function from $T \equiv\{n \in N \mid n$ has no successors $\}$ into $A \times \mathbb{R}^{I}$ that associates to each terminal node the alternative and transfers that are obtained at this node. $w^{\alpha}$ specifies the alternative, and $w^{\tau} \equiv \times_{i \in I} w_{i}^{\tau}$ specifies the transfer vector. We assume that each mechanism $m$ starts with one initial node $n_{0}$ and that the maximum length of the game tree is finite. We denote by $S_{i}(n)$ the set of strategies for $i$ at node $n$. It is possible that $\left|S_{i}(n)\right|>1$ and $\left|S_{j}(n)\right|>1$ hold for $i \neq j$, which means that

\footnotetext{
${ }^{13}$ We consider the budget-balance requirement in Section 6.1 .
} 
agents $i$ and $j$ move simultaneously at node $n$. We assume that at each node $n$, all agents know the entire history of the play.

Note that the same mechanism $m$ is always run in the mechanism stage because the social planner does not observe ex ante investments. Given realized $\theta$ and chosen ex ante investments $v$, we denote each node in the mechanism stage of the entire game by $n(\theta, v)$ if it corresponds to node $n$ of mechanism $m$. Let $N(\theta, v)(T(\theta, v)$, respectively) be the set of all corresponding nodes (terminal nodes, respectively) in mechanism $m$ in the subgame where $\theta$ is realized and $v$ is chosen.

We define a subgame-perfect equilibrium of the entire game in the following way. Let $\Xi_{i}(\theta, v)$ be the set of all mappings from $T(\theta, v)$ to $\bar{V}_{i}\left(v_{i}, \theta_{i}\right)$. Let $\mathcal{S}_{i}(\theta) \equiv V^{\theta_{i}} \times\left(\times_{v \in V^{\theta}}\right.$ $\left.\times_{n(\theta, v) \in N(\theta, v) \backslash T(\theta, v)} S_{i}(n(\theta, v))\right) \times\left(\times_{v \in V^{\theta}} \Xi_{i}(\theta, v)\right)$ be the strategy set of agent $i$ in the entire game induced by $m$ when the cost types are $\theta$. Let $\mathcal{S}(\theta) \equiv \times_{i \in I} \mathcal{S}_{i}(\theta)$. For each $v \in V^{\theta}$, $s \in \times_{i \in I} \times_{v \in V^{\theta}} \times_{n(\theta, v) \in N(\theta, v) \backslash T(\theta, v)} S_{i}(n(\theta, v))$ and $n(\theta, v) \in N(\theta, v) \backslash T(\theta, v)$, let $z(s ; n(\theta, v))$ be the terminal node when agents follow strategies $s$ in the mechanism starting from node $n(\theta, v)$.

Definition 2. A profile of strategies $\left(v^{*}, s^{*}, \xi^{*}\right) \in \mathcal{S}(\theta)$ is a subgame-perfect equilibrium (SPE) of the entire game induced by mechanism $m$ for cost types $\theta \in \Theta$ given a discount factor $\delta \in(0,1)$ if for each $i \in I$,

1. $\xi_{i}^{*}(n(\theta, v)) \in \underset{\bar{v}_{i} \in \bar{V}_{i}\left(v_{i}, \theta_{i}\right)}{\arg \max }\left\{\bar{v}_{i}\left(w^{\alpha}(n(\theta, v))\right)-c_{i}\left(\bar{v}_{i}, \theta_{i}\right)\right\}$

for any $v \in V^{\theta}$ and $n(\theta, v) \in T(\theta, v)$,

2. $s_{i}^{*} \in \underset{s_{i} \in \times_{v \in V^{\theta}} \times_{n(\theta, v) \in N(\theta, v) \backslash T(\theta, v)} S_{i}(n(\theta, v))}{\arg \max }\left\{\xi_{i}^{*}\left(z\left(s_{i}, s_{-i}^{*} ; n(\theta, v)\right)\right)\left(w^{\alpha}\left(z\left(s_{i}, s_{-i}^{*} ; n(\theta, v)\right)\right)\right)\right.$

$\left.-w_{i}^{\tau}\left(z\left(s_{i}, s_{-i}^{*} ; n(\theta, v)\right)\right)-c_{i}\left(\xi_{i}^{*}\left(z\left(s_{i}, s_{-i}^{*} ; n(\theta, v)\right)\right), \theta_{i}\right)\right\}$

for any $v \in V^{\theta}$ and $n(\theta, v) \in N(\theta, v) \backslash T(\theta, v)$,

3. $v_{i}^{*} \in \underset{v_{i} \in V^{\theta_{i}}}{\arg \max }\left\{-c_{i}\left(v_{i}, \theta_{i}\right)+\delta\left[\xi_{i}^{*}\left(z\left(s^{*} ; n_{0}\left(\theta, v_{i}, v_{-i}^{*}\right)\right)\right)\left(w^{\alpha}\left(z\left(s^{*} ; n_{0}\left(\theta, v_{i}, v_{-i}^{*}\right)\right)\right)\right)\right.\right.$

$\left.\left.-w_{i}^{\tau}\left(z\left(s^{*} ; n_{0}\left(\theta, v_{i}, v_{-i}^{*}\right)\right)\right)-c_{i}\left(\xi_{i}^{*}\left(z\left(s^{*} ; n_{0}\left(\theta, v_{i}, v_{-i}^{*}\right)\right)\right), \theta_{i}\right)+c_{i}\left(v_{i}, \theta_{i}\right)\right]\right\}$

hold. Let $\operatorname{SPE}(\theta, m, \delta) \equiv\left\{(a, t,(v, \bar{v})) \in A \times \mathbb{R}^{I} \times V^{2} \mid \exists \operatorname{SPE}(v, s, \xi)\right.$ of the entire game induced by $m$ at $\theta$ given $\delta$ s.t. $w\left(z\left(s ; n_{0}(\theta, v)\right)\right)=(a, t)$ and $\left.\xi_{i}\left(z\left(s ; n_{0}(\theta, v)\right)\right)=\bar{v}_{i} \forall i \in I\right\}$ denote the set of all outcomes and valuation functions that are on the equilibrium paths of the entire game induced by mechanism $m$ for cost types $\theta$ given a discount factor $\delta$. 
The first condition is the optimality of ex post investments. The second condition describes the SPE condition in any subgame in the mechanism stage. The third condition ensures that $v^{*}$ forms a Nash equilibrium of the ex ante investment stage.

Using the SPE of the entire game induced by the mechanism, we can define the concept of implementation in a standard way:

Definition 3. Given a discount factor $\delta \in(0,1)$, mechanism $m$ implements social choice rule $F: \Theta \rightarrow 2^{A \times \mathbb{R}^{I} \times V^{2}} \backslash\{\emptyset\}$ in subgame-perfect equilibria if $F(\theta)=S P E(\theta, m, \delta)$ holds for any profile of cost types $\theta \in \Theta$. Given a discount factor $\delta \in(0,1)$, social choice rule $F$ is implementable in subgame-perfect equilibria if there exists a mechanism that implements $F$ in subgame-perfect equilibria.

\subsection{Allocation and Transfer Rules}

Our goal is to characterize the condition for an efficient $F$ to be implementable in SPE. To do so, we focus on social choice rules $F$ for which the associated allocation and transfer rules $(\alpha, \tau)$ at the mechanism stage are functions throughout the paper. ${ }^{14}$ The associated $(\alpha, \tau)$ represents the choice of $F$ over the alternatives and transfers given the investments, and our main theorem provides a sufficient and necessary condition on $(\alpha, \tau)$ for the implementability of $F$.

To define $(\alpha, \tau)$, we first consider the valuations at the mechanism stage. When agents participate in a mechanism, the cost of ex ante investment has been sunk and each alternative should be evaluated by the optimal choice of a final valuation function.

Definition 4. The valuation function $u^{\theta_{i}, v_{i}}: A \rightarrow \mathbb{R}$ at the mechanism stage given cost type $\theta_{i} \in \Theta_{i}$ and valuation function $v_{i} \in V^{\theta_{i}}$ is defined by

$$
u^{\theta_{i}, v_{i}}(a)=\max _{\bar{v}_{i} \in \bar{V}_{i}\left(v_{i}, \theta_{i}\right)}\left\{\bar{v}_{i}(a)-c_{i}\left(\bar{v}_{i}, \theta_{i}\right)\right\}+c_{i}\left(v_{i}, \theta_{i}\right)
$$

for each $a \in A$. Let $u^{\theta, v} \equiv\left(u^{\theta_{i}, v_{i}}\right)_{i \in I}$.

This equation is taken from the second term of equation (1) and takes into account each agent's optimal ex post investment choice. Given $v_{i}$ and $a$, the optimal choice of the ex post investment $\bar{v}_{i} \in \bar{V}_{i}\left(v_{i}, \theta_{i}\right)$ maximizes $\bar{v}_{i}(a)-c_{i}\left(\bar{v}_{i}, \theta_{i}\right)$. By the compactness condition of cost

\footnotetext{
${ }^{14}$ The associated allocation and transfer correspondences exist for any implementable $F$ in general, but they are not guaranteed to be single-valued functions. We only consider functions in order to obtain a simple sufficient and necessary condition.
} 
types, we can always find an optimal $\bar{v}_{i}$. Let $U^{\theta_{i}}$ be the set of all possible valuation functions at the mechanism stage for $i$ with $\theta_{i}$, i.e., $U^{\theta_{i}} \equiv\left\{u_{i} \in \mathbb{R}^{A} \mid \exists v_{i} \in V^{\theta_{i}}\right.$ such that $\left.u^{\theta_{i}, v_{i}}=u_{i}\right\}$. Let $U^{\theta} \equiv \times_{i \in I} U^{\theta_{i}}, U_{i} \equiv \cup_{\theta_{i} \in \Theta_{i}} U^{\theta_{i}}$, and $U \equiv \times_{i \in I} U_{i}$.

An allocation rule $\alpha: U \rightarrow A$ is defined as a function that specifies an alternative for each profile of valuations at the mechanism stage. In the same way, a transfer rule $\tau: U \rightarrow \mathbb{R}^{I}$ is a function that specifies a transfer vector for each profile of valuations at the mechanism stage. $(\alpha, \tau)$ is considered to be a standard social choice function where investments are exogenously given. We say that $\alpha$ is efficient if $\alpha(u) \in \underset{a \in A}{\arg \max } \sum_{i \in I} u_{i}(a)$ for any $u \in U$.

Figure 1 describes the relationship between $F$ and $(\alpha, \tau)$. The standard Mount-Reiter diagram (without endogenous investments) is the small triangle in the bottom where $(\alpha, \tau)$ is implemented, but our problem is described by the entire triangle in which we aim to implement $F$.

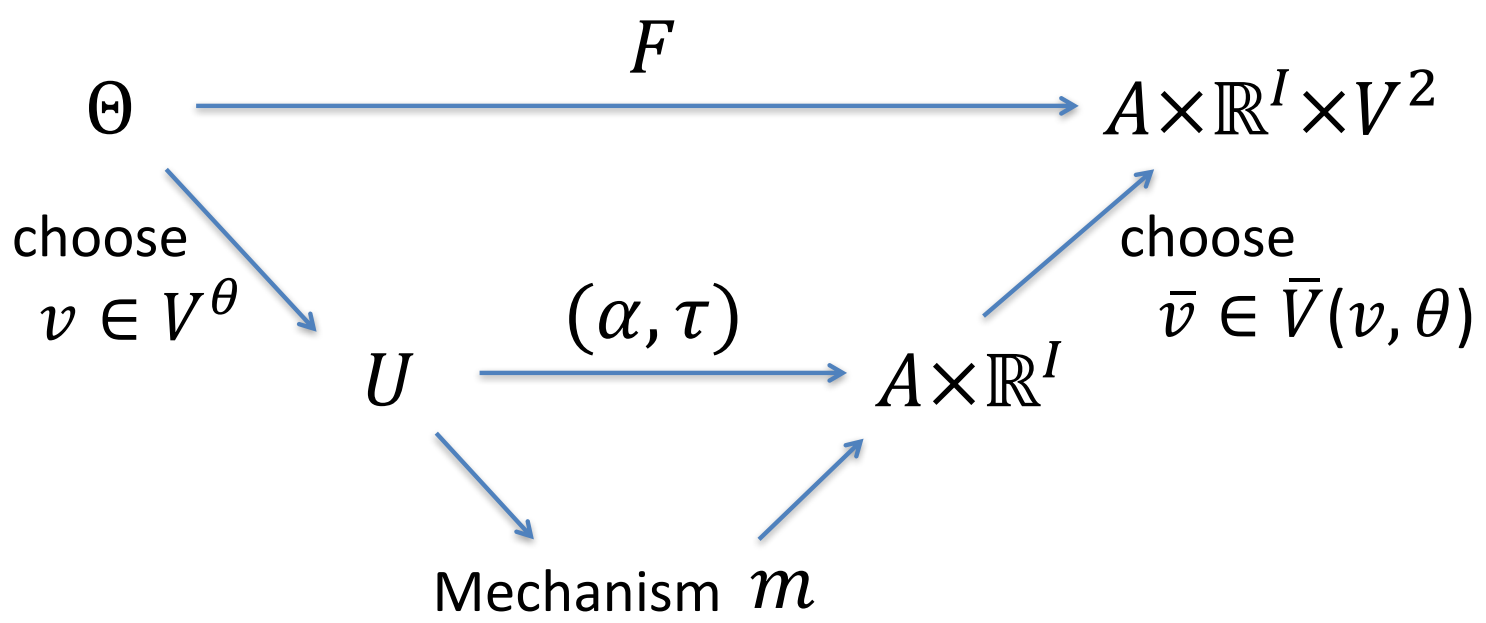

Figure 1: A social choice rule $F$ and a pair of allocation and transfer rules $(\alpha, \tau)$.

Now, we formally define the associatedness of $(\alpha, \tau)$ with $F$ :

Definition 5. A pair of allocation and transfer rules $(\alpha, \tau): U \rightarrow A \times \mathbb{R}^{I}$ is associated with a social choice rule $F: \Theta \rightarrow 2^{A \times \mathbb{R}^{I} \times V^{2}} \backslash\{\emptyset\}$ if $\alpha\left(u^{\theta, v}\right)=a$ and $\tau\left(u^{\theta, v}\right)=t$ hold for any $\theta \in \Theta$ and $(a, t,(v, \bar{v})) \in F(\theta)$.

This definition implies that for any $\theta, \theta^{\prime} \in \Theta,(a, t,(v, \bar{v})) \in F(\theta)$ and $\left(a^{\prime}, t^{\prime},\left(v^{\prime}, \bar{v}^{\prime}\right)\right) \in$ $F\left(\theta^{\prime}\right)$ with $u^{\theta, v}=u^{\theta^{\prime}, v^{\prime}},(a, t)=\left(a^{\prime}, t^{\prime}\right)$ must hold. When $F$ is efficient, we can show that the associated allocation and transfer rules $(\alpha, \tau)$ are uniquely determined and that $\alpha$ is also efficient. 
Proposition 1. Consider a social choice rule $F: \Theta \rightarrow 2^{A \times \mathbb{R}^{I} \times V^{2}} \backslash\{\emptyset\}$ and a pair of allocation and transfer rules $(\alpha, \tau): U \rightarrow A \times \mathbb{R}^{I}$ that is associated with $F$. If $F$ is efficient, $(\alpha, \tau)$ is the unique pair of associated allocation and transfer rules and $\alpha$ is efficient.

To prove this, we exploit the efficiency of $F$ and the richness of $\Theta$ : for any $u \in U$, we can find $(\theta, v)$ such that $u^{\theta, v}=u$ and $(\alpha(u), \tau(u))$ has to be determined by the information of $F$. Since Proposition 1 guarantees the uniqueness of the associated $(\alpha, \tau)$ and the efficiency of $\alpha$, our question of the implementability of an efficient $F$ essentially reduces to the design of the transfer rule $\tau$.

\section{$2.3 \quad$ Interpretation of Investments}

In our model, we assume that ex post investment is less costly than ex ante investment because of time discounting $\delta \in(0,1)$. The efficiency of $F$ implies that the efficient ex ante investment should be the one with the lowest cost (which is zero). Indeed, when ex ante investments are not possible, any efficient social choice rule can be implemented. Exploiting this, our goal is to find a condition for which no agent has an incentive to invest ex ante.

Why does the social planner not simply prohibit ex ante investment itself? In most applications such as auctions and public goods provision, the planner first announces a mechanism and there is some time period in which agents are allowed to think about their strategies in the mechanism before it is run. We argue that the agents could also strategically make some investments during this time period. In general, any kind of action that increases the valuation of a certain outcome is considered as an investment in our context. In particular, "paying a deposit" is a good example of an investment that can be done even in a short period of time. When several firms are competing for a project to be chosen by the social planner, each of them could make a contract with a relevant third party and pay a deposit to them ex ante for their preferred project. Here, a deposit is simply a prepayment of their future cost of their project, but it will not be refunded if their project is not selected by the mechanism. Then, by paying a deposit and making it a sunk cost, a firm can successfully commit to a higher profit from their project (net of the future investment cost) at the mechanism stage. This could change the outcome of the mechanism, and this firm could benefit from the ex ante deposit payment. These examples indicate that the ex ante investment that we consider in our model cannot be directly prohibited in many situations and that the agents may have an incentive for pre-mechanism investments even with $\delta \in(0,1)$. 


\section{Example}

Before showing the formal results, let us provide the intuition for why efficient investments are implemented for some transfer rules $\tau$, while they are not for others. We do so by giving numerical details to the example discussed in the Introduction section. Suppose that a city has a vacant lot and has two plans to exploit it: building either a baseball field (denoted by $a_{B}$ ) or a soccer field (denoted by $a_{S}$ ), and $A=\left\{a_{B}, a_{S}\right\} .{ }^{15}$ Either facility will be public, and all citizens will have a free access to it. Since the lot is not large enough to build both of the fields, they have to decide which one to build. There are two types of citizens: type $B$, who are interested in only playing and watching baseball, and type $S$, who are interested in only soccer. For type- $B$ citizens, the potential values of the projects are represented by $\left(v_{B}, 0\right)$, where $v_{B} \in[0,10]$ is their value for the baseball field and 0 is their value for the soccer field. Similarly, type- $S$ citizens have potential values $\left(0, v_{S}\right)$, where their value for the soccer field is denoted by $v_{S} \in[0,10]$. This means that a scalar value $v_{B}$ or $v_{S}$ is sufficient for representing their valuation functions. For simplicity, we regard each type as a representative agent and consider a problem with just two agents $B$ and $S$, i.e., $I=\{B, S\}$.

Each agent makes investments to determine her own value of the project she likes. Investment here includes several actions for them to make use of the new facility: buying equipment, collecting and organizing team members, inviting coaches for lessons, and so on. By the structure of the valuation functions in this example, investment is simply a choice of a scalar value from the interval $[0,10]$ for each agent.

We compare two efficient social choice rules, the efficient VCG social choice rule $F^{V C G}$ and the zero-transfer efficient social choice rule $F^{0} . F^{V C G}$ has the associated $\left(\alpha^{V C G}, \tau^{V C G}\right)$, which is defined as follows: for any $u=\left(u_{B}, u_{S}\right)$,

$$
\begin{aligned}
& \alpha^{V C G}(u)=\left\{\begin{array}{ll}
a_{B} & \text { if } u_{B} \geq u_{S} \\
a_{S} & \text { if } u_{B}<u_{S}
\end{array},\right. \\
& \tau_{B}^{V C G}(u)=\left\{\begin{array}{ll}
u_{S} & \text { if } u_{B} \geq u_{S} \\
0 & \text { if } u_{B}<u_{S}
\end{array},\right. \\
& \tau_{S}^{V C G}(u)=\left\{\begin{array}{ll}
0 & \text { if } u_{B} \geq u_{S} \\
u_{B} & \text { if } u_{B}<u_{S}
\end{array} .\right.
\end{aligned}
$$

Note that the domain of $(\alpha, \tau)$ is $\left\{\left(u_{B}, u_{S}\right) \in[0,10]^{2} \mid \exists \theta_{i} \in \Theta_{i}\right.$ and $v_{i} \in[0,10]$ such that $u^{\theta_{i}, v_{i}}=$ $u_{i}$ for each $\left.i=B, S\right\}$ because the valuation function at the mechanism stage can be writ-

\footnotetext{
${ }^{15}$ Suppose that the cost of building them is zero.
} 
ten by a scalar value for each agent. $\left(\alpha^{V C G}, \tau^{V C G}\right)$ is exactly the outcome function of the second-price auction in this example. ${ }^{16} F^{0}$ has the associated $\left(\alpha^{0}, \tau^{0}\right)$, which is defined as follows: for any $u=\left(u_{B}, u_{S}\right)$,

$$
\begin{aligned}
& \alpha^{0}(u)= \begin{cases}a_{B} & \text { if } u_{B} \geq u_{S} \\
a_{S} & \text { if } u_{B}<u_{S}\end{cases} \\
& \tau_{B}^{0}(u)=0, \\
& \tau_{S}^{0}(u)=0 .
\end{aligned}
$$

The allocation rule is the same as that of VCG, but no transfer is imposed under $F^{0}$.

Although the set $\Theta$ of cost types is assumed to be rich, we focus on particular cost types $\theta=\left(\theta_{B}, \theta_{S}\right)$ such that $c_{B}\left(v_{B}, \theta_{B}\right)=\frac{1}{6}\left(v_{B}\right)^{2}$ and $c_{S}\left(v_{S}, \theta_{S}\right)=\frac{1}{4}\left(v_{S}\right)^{2}$. First, consider $F^{V C G}(\theta)$ and $F^{0}(\theta)$. Since both social choice rules are efficient, the alternative and investments should be the same. By $\delta<1$, efficient ex ante investments should be zero, i.e., $v_{B}=v_{S}=0$. If a baseball field is built, the optimal ex post investment of $B$ should be $\underset{\bar{v}_{B} \in[0,10]}{\arg \max }\left\{-\frac{1}{6}\left(\bar{v}_{B}\right)^{2}+\right.$ $\left.\bar{v}_{B}\right\}=3$. As $S^{\prime}$ 's value for the baseball field is always zero, the maximum social welfare in this case is $\frac{3}{2} \delta$. Similarly, if the soccer field is built, the optimal investment of $S$ should be $\underset{\bar{v}_{S} \in[0,10]}{\arg \max }\left\{-\frac{1}{4}\left(\bar{v}_{S}\right)^{2}+\bar{v}_{S}\right\}=2$, and the maximum social welfare in this case is $\delta$. Therefore, for each $F \in\left\{F^{V C G}, F^{0}\right\}$ and any $\left(a^{*}, t^{*},\left(v^{*}, \bar{v}^{*}\right)\right) \in F(\theta)$, we have $a^{*}=a_{B},\left(v_{B}^{*}, \bar{v}_{B}^{*}\right)=(0,3)$ and $\left(v_{S}^{*}, \bar{v}_{S}^{*}\right)=(0,0)$.

For each $F \in\left\{F^{V C G}, F^{0}\right\}$, we examine whether $F(\theta)=S P E(\theta, m, \delta)$ holds for some mechanism $m$. Since each $F$ has the associated $(\alpha, \tau)$, if there exists a mechanism $m$ that implements $F$ in SPE, the SPE outcomes of $m$ in the mechanism stage should be consistent with $(\alpha, \tau)$. Note that for any $(\alpha, \tau)$, we can find a mechanism $m$ that implements $(\alpha, \tau)$ in the mechanism stage (Moore and Repullo, 1988). Let $m^{V C G}\left(m^{0}\right.$, respectively) be the mechanism that implements $\left(\alpha^{V C G}, \tau^{V C G}\right)\left(\left(\alpha^{0}, \tau^{0}\right)\right.$, respectively). Then, the entire game induced by each $m \in\left\{m^{V C G}, m^{0}\right\}$ is written as follows:

1. Each agent $i=B, S$ simultaneously chooses $v_{i}$ from $[0,10]$. Each $i$ incurs the cost of investment $c_{i}\left(v_{i}, \theta_{i}\right)$.

2. Mechanism $m$ implements the allocation and transfers rules $(\alpha, \tau)$ for their valuations $\left(u^{\theta_{B}, v_{B}}, u^{\theta_{S}, v_{S}}\right)$.

3. Each agent $i=B, S$ chooses the final valuation $\bar{v}_{i}$ from $\left[v_{i}, 10\right]$. Each $i$ incurs the cost of additional investment $c_{i}\left(\bar{v}_{i}, \theta_{i}\right)-c_{i}\left(v_{i}, \theta_{i}\right)$.

\footnotetext{
${ }^{16}$ The choice of a tie-breaking rule does not matter for the results in this example.
} 
The ex ante utility of agent $i=B, S$ is

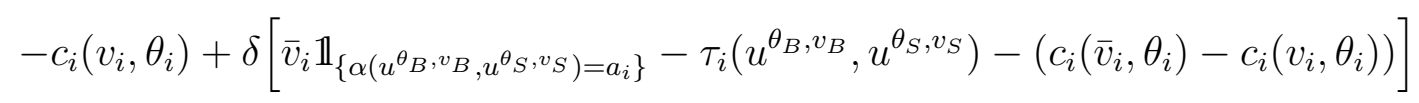

where $j$ is the other agent and $\mathbb{1}$ is the indicator function. ${ }^{17}$

$\mathrm{SPE}$ is solved by backward induction. Consider $B$ 's optimal ex post investment given $v_{B}$ and the chosen project. Since $B$ makes further investment only when the baseball field is built and $v_{B}$ is less than 3 , the optimal $\bar{v}_{B}$ given $v_{B}$ is

$$
\bar{v}_{B}= \begin{cases}\max \left\{3, v_{B}\right\} & \text { if } \alpha\left(u^{\theta_{B}, v_{B}}, u^{\theta_{S}, v_{S}}\right)=a_{B} \\ v_{B} & \text { otherwise. }\end{cases}
$$

Similarly, $S$ 's optimal $\bar{v}_{S}$ given $v_{S}$ and the chosen project is

$$
\bar{v}_{S}= \begin{cases}\max \left\{2, v_{S}\right\} & \text { if } \alpha\left(u^{\theta_{B}, v_{B}}, u^{\theta_{S}, v_{S}}\right)=a_{S} \\ v_{S} & \text { otherwise. }\end{cases}
$$

Next, let us compute the valuations $\left(u^{\theta_{B}, v_{B}}, u^{\theta_{S}, v_{S}}\right)$ at the mechanism stage for each $\left(v_{B}, v_{S}\right) \in[0,10]^{2}$. Following Definition 4 , the valuations are computed as

$$
u^{\theta_{B}, v_{B}}=\max _{\bar{v}_{B} \in\left[v_{B}, 10\right]}\left\{\bar{v}_{B}-\left(c_{B}\left(\bar{v}_{B}, \theta_{B}\right)-c_{B}\left(v_{B}, \theta_{B}\right)\right)\right\}= \begin{cases}\frac{3}{2}+\frac{1}{6}\left(v_{B}\right)^{2} & \text { if } v_{B} \in[0,3) \text { and } \\ v_{B} & \text { if } v_{B} \in[3,10],\end{cases}
$$

and

$$
u^{\theta_{S}, v_{S}}=\max _{\bar{v}_{S} \in\left[v_{S}, 10\right]}\left\{\bar{v}_{S}-\left(c_{S}\left(\bar{v}_{S}, \theta_{S}\right)-c_{S}\left(v_{S}, \theta_{S}\right)\right)\right\}= \begin{cases}1+\frac{1}{4}\left(v_{S}\right)^{2} & \text { if } v_{S} \in[0,2) \text { and } \\ v_{S} & \text { if } v_{S} \in[2,10] .\end{cases}
$$

Intuitively, when $v_{i}$ is higher than the optimal value, $u^{\theta_{i}, v_{i}}$ is equal to $v_{i}$ as there is no further investment. If $v_{i}$ is lower than the optimal value, $u^{\theta_{i}, v_{i}}$ is increasing in $v_{i}$ exactly by the amount of $c_{i}\left(v_{i}, \theta_{i}\right)$ because more ex ante investment means that there is a smaller cost for additional investment when $i$ 's favorite project is realized.

Finally, we analyze the ex ante investment stage of the entire game.

[1] Ex ante investments induced by $m^{V C G}$.

\footnotetext{
${ }^{17}$ For any proposition $p, \mathbb{1}_{\{p\}}$ is defined by

$$
\mathbb{1}= \begin{cases}1 & \text { if } p \text { is true } \\ 0 & \text { otherwise }\end{cases}
$$
}


Consider $S$ 's incentive. If the soccer field is built, $S$ 's VCG payment is at least $\frac{3}{2}$ because $u^{\theta_{B}, v_{B}} \geq \frac{3}{2}$ holds for any $v_{B} \in[0,10]$. However, since the net utility from the soccer project cannot exceed 1 for $S$, she does not have an incentive to realize $a_{S}$. That is,

$$
-(1-\delta) \frac{1}{4}\left(v_{S}\right)^{2}+\delta\left(\max \left\{2, v_{S}\right\}-u^{\theta_{B}, v_{B}}-\frac{1}{4}\left(\max \left\{2, v_{S}\right\}\right)^{2}\right)<0
$$

for any $\left(v_{B}, v_{S}\right) \in[0,10]^{2}$ with $u^{\theta_{S}, v_{S}} \geq u^{\theta_{B}, v_{B}}$ and $\delta \in(0,1)$. Given $v_{S}=0$, B's SPE strategy also leads to the efficient investments $\left(v_{B}^{*}, \bar{v}_{B}^{*}\right)=(0,3)$. Thus, $F^{V C G}(\theta)=\operatorname{SPE}\left(\theta, m^{V C G}, \delta\right)$ holds for these cost types $\theta$.

[2] Ex ante investments induced by $m^{0}$.

Now, $S$ has an incentive to invest more than $B$ as long as $B$ 's investment is efficient, i.e., $v_{B}=0$. This occurs because the payment in the mechanism is always zero and $S$ would still earn positive utility from the soccer project. That is, there exists $v_{S} \in\left(\frac{3}{2}, 4\right)$ and $\delta \in(0,1)$ such that

$$
-(1-\delta) \frac{1}{4}\left(v_{S}\right)^{2}+\delta\left(\max \left\{2, v_{S}\right\}-0-\frac{1}{4}\left(\max \left\{2, v_{S}\right\}\right)^{2}\right)>0 .
$$

Indeed, there is a mixed strategy equilibrium in which $v_{S}>0$ occurs with a positive probability. Thus, $F^{0}(\theta) \neq S P E\left(\theta, m^{0}, \delta\right)$ holds for these cost types $\theta$.

This example illustrates that the transfer rule is crucial for inducing the right incentive for $S$. Suppose that $B$ chooses $v_{B}=0$, and consider two options for $S: v_{S}=0$ or 2 . The baseball project will be realized in the former case, but $S$ could realize the soccer project in the latter case. To prevent $S$ from investing, the transfer rule $\tau$ for $\left(\frac{3}{2}, 2\right)$ should satisfy

$$
0 \geq-c_{S}\left(2, \theta_{S}\right)+\delta\left\{u^{\theta_{S}, 2}-\tau_{S}\left(\frac{3}{2}, 2\right)\right\} \Leftrightarrow \tau_{S}\left(\frac{3}{2}, 2\right) \geq 2-\frac{1}{\delta}
$$

$\tau^{V C G}$ satisfies this condition for any $\delta \in(0,1)$, but $\tau^{0}$ does not for $\delta \in\left(\frac{1}{2}, 1\right)$. Moreover, we can see that $\tau^{V C G}$ is not the unique transfer rule that satisfies this inequality. Our main theorem generalizes this idea and finds a sufficient and necessary condition on $(\alpha, \tau)$ for an efficient $F$ to be implementable.

\section{Main Results}

\subsection{Commitment-proofness}

First, we formally define the main concept of our paper. 
Definition 6. A pair of allocation and transfer rules $(\alpha, \tau)$ is commitment-proof if for any $i \in I, u \in U$ and $\tilde{u}_{i} \in U_{i}$,

$$
\tilde{u}_{i}\left(\alpha\left(\tilde{u}_{i}, u_{-i}\right)\right)-\tau_{i}\left(\tilde{u}_{i}, u_{-i}\right)-\max \left\{0, \max _{a \in A}\left\{\tilde{u}_{i}(a)-u_{i}(a)\right\}\right\} \leq u_{i}(\alpha(u))-\tau_{i}(u) .
$$

Note that this is a condition on the allocation and transfer rules $(\alpha, \tau)$ and does not involve the investment structure of our model. To interpret this condition, consider the following situation: (i) $(\alpha, \tau)$ is implemented, (ii) the agents are assigned their "default" valuation functions $u \in U$, and (iii) each agent $i$ could change $u_{i}$ to any other valuation functions in $U_{i}$ ex ante through a certain costly commitment device. The RHS of equation (2) is agent $i$ 's default utility from $(\alpha, \tau)$. Since agents would arbitrarily increase their valuations if the commitment device were free, suppose that the cost of commitment from $u_{i}$ to $\tilde{u}_{i}$ is $\max \left\{0, \max _{a \in A}\left\{\tilde{u}_{i}(a)-u_{i}(a)\right\}\right\}$. Then, $i$ 's utility from the commitment to $\tilde{u}_{i}$ is represented by the LHS of equation (2). The equation (2) requires that from any default valuation functions, none of the agents should have an incentive to commit to any other valuation function through this commitment device.

The commitment-proofness condition can be written in the following form as well:

$$
u_{i}\left(\alpha\left(\tilde{u}_{i}, u_{-i}\right)\right)-\tau_{i}\left(\tilde{u}_{i}, u_{-i}\right)-\epsilon\left(u, \tilde{u}_{i}, \alpha\right) \leq u_{i}(\alpha(u))-\tau_{i}(u)
$$

where $\epsilon\left(u, \tilde{u}_{i}, \alpha\right)=\max \left\{0, \max _{a \in A}\left\{\tilde{u}_{i}(a)-u_{i}(a)\right\}\right\}-\left[\tilde{u}_{i}\left(\alpha\left(\tilde{u}_{i}, u_{-i}\right)\right)-u_{i}\left(\alpha\left(\tilde{u}_{i}, u_{-i}\right)\right)\right]$. Since $\epsilon\left(u, \tilde{u}_{i}, \alpha\right) \geq 0$ always holds, we can see that commitment-proofness is implied by strategyproofness: $u_{i}\left(\alpha\left(\tilde{u}_{i}, u_{-i}\right)\right)-\tau_{i}\left(\tilde{u}_{i}, u_{-i}\right) \leq u_{i}(\alpha(u))-\tau_{i}(u)$ for any $i \in I, u \in U$ and $\tilde{u}_{i} \in U_{i}$. However, this is not a standard approximation of strategy-proofness because $\epsilon$ is not a given value but rather depends on the valuation functions $u, \tilde{u}_{i}$ and the allocation rule $\alpha$. $\epsilon\left(u, \tilde{u}_{i}, \alpha\right)$ can become zero or a large positive value. To illustrate the key feature of commitment-proofness, we provide an example of $(\alpha, \tau)$ that is commitment-proof but not strategy-proof.

Example 1. Suppose $I=\{i, j\}$ and $A=\left\{a_{1}, a_{2}, a_{3}\right\}$. Consider the following set of valuation functions $U$ :

$$
\begin{aligned}
& U_{i}=\left\{u_{i}, \tilde{u}_{i}\right\} \\
& U_{j}=\left\{u_{j}\right\}
\end{aligned}
$$

such that

\begin{tabular}{c|c|c|c} 
& $a_{1}$ & $a_{2}$ & $a_{3}$ \\
\hline \hline$u_{i}(\cdot)$ & 0 & 2 & 0 \\
\hline$\tilde{u}_{i}(\cdot)$ & 1 & 5 & 5 \\
\hline$u_{j}(\cdot)$ & 5 & 2 & 0
\end{tabular}


Consider the following $(\alpha, \tau): \alpha$ is efficient; that is,

$$
\alpha\left(u_{i}, u_{j}\right)=a_{1}, \alpha\left(\tilde{u}_{i}, u_{j}\right)=a_{2},
$$

and $\tau$ satisfies

$$
\tau_{i}\left(u_{i}, u_{j}\right)=0, \tau_{i}\left(\tilde{u}_{i}, u_{j}\right)=1
$$

Let us examine the strategy-proofness and commitment-proofness conditions for agent $i$. First, since

$$
u_{i}\left(\alpha\left(\tilde{u}_{i}, u_{j}\right)\right)-\tau_{i}\left(\tilde{u}_{i}, u_{j}\right)=2-1>0-0=u_{i}(\alpha(u))-\tau_{i}(u)
$$

$(\alpha, \tau)$ is not strategy-proof. On the other hand, $(\alpha, \tau)$ is commitment-proof because

$\tilde{u}_{i}\left(\alpha\left(\tilde{u}_{i}, u_{j}\right)\right)-\tau_{i}\left(\tilde{u}_{i}, u_{j}\right)-\max \left\{0, \max _{a \in A}\left\{\tilde{u}_{i}(a)-u_{i}(a)\right\}\right\}=5-1-5 \leq 0-0=u_{i}(\alpha(u))-\tau_{i}(u)$

and

$u_{i}(\alpha(u))-\tau_{i}(u)-\max \left\{0, \max _{a \in A}\left\{u_{i}(a)-\tilde{u}_{i}(a)\right\}\right\}=0-0-0 \leq 5-1=\tilde{u}_{i}\left(\alpha\left(\tilde{u}_{i}, u_{j}\right)\right)-\tau_{i}\left(\tilde{u}_{i}, u_{j}\right)$.

The deviation from $u_{i}$ to $\tilde{u}_{i}$ highlights the difference between these two concepts. We can also see that this $(\alpha, \tau)$ satisfies commitment-proofness using the alternative definition:

$$
u_{i}\left(\alpha\left(\tilde{u}_{i}, u_{-i}\right)\right)-\tau_{i}\left(\tilde{u}_{i}, u_{-i}\right)-\epsilon\left(u, \tilde{u}_{i}, \alpha\right)=2-1-2 \leq 0-0=u_{i}(\alpha(u))-\tau_{i}(u) .
$$

Here, $\underset{a \in A}{\arg \max }\left\{\tilde{u}_{i}(a)-u_{i}(a)\right\}=a_{3} \neq a_{2}=\alpha\left(\tilde{u}_{i}, u_{j}\right)$ implies $\epsilon\left(u, \tilde{u}_{i}, \alpha\right)>0$, and this allows the non-strategy-proof $(\alpha, \tau)$ to satisfy commitment-proofness.

\subsection{Implementability of Efficient $F$}

For our main theorem, the following lemma is useful.

Lemma 1. For any agent $i \in I$ and any cost type $\theta_{i} \in \Theta_{i}$,

$$
c_{i}\left(v_{i}, \theta_{i}\right) \geq \max _{a \in A}\left\{u^{\theta_{i}, v_{i}}(a)-u^{\theta_{i}, v_{i}^{0}}(a)\right\}
$$

holds for any $v_{i} \in V^{\theta_{i}}$ and $v_{i}^{0} \in Z^{\theta_{i}}\left(=\left\{\tilde{v}_{i} \in V^{\theta_{i}} \mid c_{i}\left(\tilde{v}_{i}, \theta_{i}\right)=0\right\}\right)$.

Proof: According to the definition of the valuation at the mechanism stage,

$$
\begin{aligned}
u^{\theta_{i}, v_{i}^{0}}(a) & =\max _{\bar{v}_{i} \in V^{\theta_{i}}}\left\{\bar{v}_{i}(a)-c_{i}\left(\bar{v}_{i}, \theta_{i}\right)\right\} \\
& \geq \max _{\bar{v}_{i} \in \bar{V}_{i}\left(v_{i}, \theta_{i}\right)}\left\{\bar{v}_{i}(a)-c_{i}\left(\bar{v}_{i}, \theta_{i}\right)\right\} \\
& =u^{\theta_{i}, v_{i}}(a)-c_{i}\left(v_{i}, \theta_{i}\right)
\end{aligned}
$$


holds for any $a \in A$. Thus, we have $c_{i}\left(v_{i}, \theta_{i}\right) \geq \max _{a \in A}\left\{u^{\theta_{i}, v_{i}}(a)-u^{\theta_{i}, v_{i}^{0}}(a)\right\}$.

This lemma shows that the cost of any ex ante investment $v_{i}$ is at least as high as the maximal increment from $u^{\theta_{i}, v_{i}^{0}}$ with least costly ex ante investment $v_{i}^{0}$ to $u^{\theta_{i}, v_{i}}$. The following theorem is our main result, which identifies the condition for an efficient $F$ to be implementable.

Theorem 1. Consider an efficient social choice rule $F: \Theta \rightarrow 2^{A \times \mathbb{R}^{I} \times V^{2}} \backslash\{\emptyset\}$ and a pair of allocation and transfer rules $(\alpha, \tau): U \rightarrow A \times \mathbb{R}^{I}$ that is associated with $F$. F is implementable in subgame-perfect equilibria for any discount factor $\delta \in(0,1)$ if and only if $(\alpha, \tau)$ is commitment-proof.

The key idea of this theorem is to apply the commitment-proof condition to the choice between the least costly investment $v_{i}^{0} \in Z^{\theta_{i}}$ and any other investment $v_{i} \in V^{\theta_{i}}$ in the ex ante investment stage. Lemma 1 implies that $\left(u^{\theta_{i}, v_{i}^{0}}, u^{\theta_{i}, v_{i}}, c_{i}\left(v_{i}, \theta_{i}\right)\right)$ corresponds to $\left(u_{i}, \tilde{u}_{i}, \max \left\{0, \max _{a \in A}\left\{\tilde{u}_{i}(a)-u_{i}(a)\right\}\right\}\right)$ in the definition of commitment-proofness. Then, in the if part of the proof, we show that $i$ has a dominant strategy to choose $v_{i}^{0}$ ex ante when $(\alpha, \tau)$ is commitment-proof, which leads to the implementation of efficient investments. Moreover, we show the necessity of commitment-proofness by exploiting the richness of the set $\Theta$ of cost types. Namely, whenever $(\alpha, \tau)$ is not commitment-proof for $i$, we can find a cost type for which $i$ has an incentive to make a costly ex ante investment $v_{i} \in V^{\theta_{i}} \backslash Z^{\theta_{i}}$, which leads to the inefficiency of this SPE. Therefore, we conclude that for an efficient $F$ to be implementable, commitment-proofness of the associated $(\alpha, \tau)$ is sufficient and necessary.

The main contribution of this theorem is that it provides a condition on the associated $(\alpha, \tau)$ for an efficient $F$ to be implementable. Since our social choice rule $F$ is not standard in the sense that non-contractible investments are included as the outcomes of $F$, deriving a condition on $(\alpha, \tau)$, which is a standard social choice function given investments, is useful for the social planner who designs a mechanism. Since Proposition 1 shows that the associated $\alpha$ is always efficient for efficient $F$, the commitment-proofness condition matters for the design of $\tau$. We also know that the set of commitment-proof and efficient $(\alpha, \tau)$ is non-empty as commitment-proofness is weaker than strategy-proofness.

Although the sufficiency of commitment-proofness holds for any $\delta$ that is arbitrarily close to one, it does not for $\delta=1 .^{18}$ Intuitively, when $\delta$ is one, investing ex ante and ex post can be indifferent and there exists an equilibrium in which more than one agents invest ex ante. The next example illustrates this using the efficient $V C G$ social choice rule $F^{V C G}$. $F^{V C G}$ is

\footnotetext{
${ }^{18}$ Note that the necessity of commitment-proofness in Theorem 1 still holds for $\delta=1$.
} 
efficient in the sense of Definition 1 , and the associated $\left(\alpha^{V C G}, \tau^{V C G}\right)$ is the standard VCG function, which is strategy-proof: for any $u \in U$,

$$
\begin{aligned}
& \alpha^{V C G}(u) \in \underset{a \in A}{\arg \max } \sum_{i \in I} u_{i}(a), \\
& \tau_{i}^{V C G}(u)=\max _{a \in A} \sum_{j \in I \backslash\{i\}} u_{j}(a)-\sum_{j \in I \backslash\{i\}} u_{j}\left(\alpha^{V C G}(u)\right) \text { for any } i \in I .
\end{aligned}
$$

Observation 1. The efficient VCG social choice rule $F^{V C G}$ is not implementable in SPE when $\delta=1$.

Example 2. Let $\{i, j\} \subseteq I$ and $\left\{a_{1}, a_{2}\right\} \subseteq A$. Consider the following cost types $\theta$ :

$$
\begin{aligned}
V^{\theta_{i}} & =\left\{u_{i}, \tilde{u}_{i}\right\}, \\
V^{\theta_{j}} & =\left\{u_{j}, \tilde{u}_{j}\right\}, \\
V^{\theta_{k}} & =\{0\} \text { for any } k \in I \backslash\{i, j\},
\end{aligned}
$$

where

$$
\begin{aligned}
& u_{i}\left(a_{1}\right)=u_{j}\left(a_{1}\right)=5, \quad u_{i}\left(a_{2}\right)=u_{j}\left(a_{2}\right)=4, u_{i}(a)=u_{j}(a)=0 \text { for any } a \in A \backslash\left\{a_{1}, a_{2}\right\}, \\
& \tilde{u}_{i}\left(a_{1}\right)=\tilde{u}_{j}\left(a_{1}\right)=0, \tilde{u}_{i}\left(a_{2}\right)=\tilde{u}_{j}\left(a_{2}\right)=6, \tilde{u}_{i}(a)=\tilde{u}_{j}(a)=0 \text { for any } a \in A \backslash\left\{a_{1}, a_{2}\right\},
\end{aligned}
$$

and

$$
\begin{aligned}
& c_{i}\left(u_{i}, \theta_{i}\right)=c_{j}\left(u_{j}, \theta_{j}\right)=0, \\
& c_{i}\left(\tilde{u}_{i}, \theta_{i}\right)=c_{j}\left(\tilde{u}_{j}, \theta_{j}\right)=2, \\
& c_{k}\left(0, \theta_{k}\right)=0 \text { for any } k \in I \backslash\{i, j\} .
\end{aligned}
$$

We only need to consider agents $i$ and $j$. Since the maximum social welfare is $\delta(5+5)=10$, efficient ex ante investments of $i$ and $j$ in $F^{V C G}(\theta)$ should be $\left(u_{i}, u_{j}\right)$.

Suppose, for the sake of contradiction, that there exists a mechanism $m$ that implements $F^{V C G}$ in SPE. Then, the outcome of $m$ has to be consistent with $\left(\alpha^{V C G}, \tau^{V C G}\right)$. Consider the entire game induced by $m$. We first examine the optimal ex post investment for $i$ (note that $i$ and $j$ are symmetric). If $i$ chooses $\tilde{u}_{i}$ as an ex ante investment, since $c_{i}\left(\tilde{u}_{i}, \theta_{i}\right)>c_{i}\left(u_{i}, \theta_{i}\right)$, the ex post investment must be $\tilde{u}_{i}$. The valuation at the mechanism stage is also $\tilde{u}_{i}$. When $i$ chooses $u_{i}$ as an ex ante investment, since $u_{i}(a) \geq \tilde{u}_{i}(a)-c_{i}\left(\tilde{u}_{i}, \theta_{i}\right)$ holds for any $a \in A$, the ex post investment and the valuation at the mechanism stage are both $u_{i}$.

Next, suppose that $j$ chooses $\tilde{u}_{j}$ in the ex ante investment stage. If $i$ chooses $u_{i}$, the SPE outcome of $m$ should be $\alpha^{V C G}\left(u_{i}, \tilde{u}_{j}, 0\right)=a_{2}$ and $\tau_{i}^{V C G}\left(u_{i}, \tilde{u}_{j}, 0\right)=0$. The ex ante utility of $i$ is $4 \delta=4$. On the other hand, when $i$ chooses $\tilde{u}_{i}$, the SPE outcome will be 
$\alpha^{V C G}\left(\tilde{u}_{i}, \tilde{u}_{j}, 0\right)=a_{2}$ and $\tau_{i}^{V C G}\left(\tilde{u}_{i}, \tilde{u}_{j}, 0\right)=0$, and the ex ante utility of agent $i$ is $6 \delta-2=4$. Thus, there is an SPE in which $i$ and $j$ choose $\left(\tilde{u}_{i}, \tilde{u}_{j}\right)$ as ex ante investments. However, this is a contradiction because these SPE ex ante investments $\left(\tilde{u}_{i}, \tilde{u}_{j}\right)$ achieve the social welfare of 8 , which is not the outcome of $F^{V C G}$.

\section{Without Ex Post Investments}

\subsection{Relation to Rogerson (1992) and Hatfield et al. (2018)}

In our main model, although the set $\Theta_{i}$ of cost types is rich, there is a natural restriction on the set of valuations at the mechanism stage and their costs because of the optimality of ex post investment. To see this, recall that the set of valuations at the mechanism stage for type $\theta_{i}$ is given by $U^{\theta_{i}} \equiv\left\{u_{i} \in \mathbb{R}^{A} \mid \exists v_{i} \in V^{\theta_{i}}\right.$ such that $\left.u^{\theta_{i}, v_{i}}=u_{i}\right\}$. If we see our model as a one-shot ex ante investment choice problem, an agent with type $\theta_{i}$ faces a choice of valuations $u_{i}=u^{\theta_{i}, v_{i}} \in U^{\theta_{i}}$ for which the costs are given by $c_{i}\left(v_{i}, \theta_{i}\right)$. The key restriction between $u^{\theta_{i}, v_{i}}$ and $c_{i}\left(v_{i}, \theta_{i}\right)$ is represented by Lemma 1 .

On the other hand, in many papers such as Rogerson (1992) and Hatfield et al. (2018), such restrictions on the set of valuations and their costs are not imposed because ex post investment is not explicitly modeled. These studies typically obtain results on the existence of an efficient investment equilibrium, which are more conservative than our Theorem 1 . In particular, Hatfield et al. (2018) showed that for allocatively efficient mechanisms, strategyproofness is sufficient and necessary for the existence of an ex ante efficient investment equilibrium. The necessity part of their theorem exploits the assumption that any valuation (at the mechanism stage) could be chosen at any cost. Here, a natural question is the following: What happens to our full implementation problem when no ex post investment is possible but the set of cost types is still rich?

\subsection{Impossibility Result}

By extending the logic of Hatfield et al. (2018), we prove the following impossibility result: without ex post investments, there does not exist any social choice rule that is efficient and implementable in subgame-perfect equilibria.

To do so, we first redefine several concepts in this environment accordingly. We consider a special case of social choice rules $G: \Theta \rightarrow 2^{A \times \mathbb{R}^{I} \times V} \backslash\{\emptyset\}$, where only ex ante investments are specified. As in the main part of the model, we only consider $G$ whose associated allocation and transfer rules are functions: the associated allocation and transfer rules are defined as 
$(\alpha, \tau): V \rightarrow A \times \mathbb{R}^{I}$ such that $\alpha(v)=a$ and $\tau(v)=t$ for any $\theta \in \Theta$ and $(a, t, v) \in G(\theta)$. Here, we assume $\delta=1$ without loss of generality because there is only one investment stage. The efficiency condition becomes simpler: for any $\theta \in \Theta$,

$\left\{\left(a^{*}, v^{*}\right) \in A \times V \mid\left(a^{*}, t^{*}, v^{*}\right) \in G(\theta)\right.$ for some $\left.t^{*} \in \mathbb{R}^{I}\right\}=\underset{(a, v) \in A \times V}{\arg \max } \sum_{i \in I}\left\{-c_{i}\left(v_{i}, \theta_{i}\right)+v_{i}(a)\right\}$.

We consider the same definition of mechanisms, but the entire game reduces to the entire game without ex post investments induced by a mechanism, where agents cannot make further investments after the mechanism stage. Let $\tilde{\mathcal{S}}_{i}(\theta) \equiv V^{\theta_{i}} \times\left(\times_{v \in V^{\theta}} \times_{n(\theta, v) \in N(\theta, v) \backslash T(\theta, v)} S_{i}(n(\theta, v))\right)$ be the strategy set of agent $i$ in this game when the cost types are $\theta$. Let $\tilde{\mathcal{S}}(\theta) \equiv \times_{i \in I} \tilde{\mathcal{S}}_{i}(\theta)$. In this setting, SPE and the implementability of $G$ are defined in the following way:

Definition 7. A profile of strategies $\left(v^{*}, s^{*}\right) \in \tilde{\mathcal{S}}(\theta)$ is a subgame-perfect equilibrium (SPE) of the entire game without ex post investments induced by mechanism $m$ for cost types $\theta \in \Theta$ if for each $i \in I$,

1. $s_{i}^{*} \in$

$$
\underset{s_{i} \in \times_{v \in V^{\theta}} \times_{n(\theta, v) \in N(\theta, v) \backslash T(\theta, v)} S_{i}(n(\theta, v))}{\arg \max }\left\{v_{i}\left(w^{\alpha}\left(z\left(s_{i}, s_{-i}^{*} ; n(\theta, v)\right)\right)\right)-w_{i}^{\tau}\left(z\left(s_{i}, s_{-i}^{*} ; n(\theta, v)\right)\right)\right\}
$$

for any $v \in V^{\theta}$ and $n(\theta, v) \in N(\theta, v) \backslash T(\theta, v)$,

2. $v_{i}^{*} \in \underset{v_{i} \in V^{\theta_{i}}}{\arg \max }\left\{-c_{i}\left(v_{i}, \theta_{i}\right)+v_{i}\left(w^{\alpha}\left(z\left(s^{*} ; n_{0}\left(\theta, v_{i}, v_{-i}^{*}\right)\right)\right)\right)-w_{i}^{\tau}\left(z\left(s^{*} ; n_{0}\left(\theta, v_{i}, v_{-i}^{*}\right)\right)\right)\right\}$

hold. Let $\operatorname{SPE}(\theta, m) \equiv\left\{(a, t, v) \in A \times \mathbb{R}^{I} \times V \mid \exists \operatorname{SPE}(v, s)\right.$ of the entire game without ex post investments induced by $m$ at $\theta$ s.t. $\left.w\left(z\left(s ; n_{0}(\theta, v)\right)\right)=(a, t)\right\}$ denote the set of all outcomes and valuation functions that are on the equilibrium paths of the entire game without ex post investments induced by mechanism $m$ for cost types $\theta$.

Definition 8. Mechanism $m$ implements social choice rule $G: \Theta \rightarrow 2^{A \times \mathbb{R}^{I} \times V} \backslash\{\emptyset\}$ in subgame-perfect equilibria without ex post investments if $G(\theta)=S P E(\theta, m)$ holds for any profile of cost types $\theta \in \Theta$. Social choice rule $G$ is implementable in subgame-perfect equilibria without ex post investments if there exists a mechanism that implements $G$ in subgameperfect equilibria.

The next proposition is the impossibility result in this setting.

Proposition 2. There does not exist a social choice rule $G: \Theta \rightarrow 2^{A \times \mathbb{R}^{I} \times V} \backslash\{\emptyset\}$ that is efficient and implementable in subgame-perfect equilibria without ex post investments. 
First, it is easy to prove the counterpart of Proposition 1 in this setting. That is, the pair of associated allocation and transfer rules $(\alpha, \tau): V \rightarrow A \times \mathbb{R}^{I}$ has to be unique, and the efficiency of $G$ implies that $\alpha$ must be efficient in a standard sense: given any $v \in V$, $\alpha(v) \in \underset{a \in A}{\arg \max } \sum_{i \in I} v_{i}(a)$.

Then, we consider the following two cases: when $(\alpha, \tau)$ is strategy-proof and when it is not. When $(\alpha, \tau)$ is not strategy-proof, the logic follows from the necessity of strategyproofness for the existence of an efficient investment equilibrium (Hatfield et al., 2018). Since there is no ex post investment, the valuation functions at the mechanism stage can be associated with any investment costs because of the richness condition. Therefore, we can find cost types at which the privately optimal ex ante investment choice for some agent does not maximize the social welfare.

On the other hand, for any efficient and strategy-proof $(\alpha, \tau)$, we can always find cost types for which an inefficient investment equilibrium exists in addition to the efficient one. Basically, we embed the example in Section 3 to any general problem and show the multiplicity of SPE. This is possible because given efficiency and strategy-proofness, the transfer rule $\tau$ is unique up to a constant.

\subsection{Example Revisited}

To illustrate the main idea of Proposition 2 for the case of strategy-proof $(\alpha, \tau)$, we revisit the two-alternative example in Section 3.

Recall that there are two agents, $B$ and $S$, and each of them is interested only in project $a_{i}$ with $i=B, S$. Suppose, for the sake of contradiction, that $F^{V C G}$ can be implemented by mechanism $m$ in SPE, and consider the entire game without ex post investments induced by $m$. Since $\left(\alpha^{V C G}, \tau^{V C G}\right)$ must be the unique pair of the associated allocation and transfer rules as shown in Proposition 2, the entire game is written as follows:

1. Each agent $i=B, S$ simultaneously chooses $v_{i}$ from $[0,10]$. Each $i$ incurs the cost of investment $c_{i}\left(v_{i}, \theta_{i}\right)$.

2. The mechanism $m$ implements $\left(\alpha^{V C G}, \tau^{V C G}\right)$ for their valuations $\left(v_{B}, v_{S}\right)$.

Given $\left(\alpha^{V C G}, \tau^{V C G}\right)$, the ex ante utility of agent $i=B, S$ for $v=\left(v_{B}, v_{S}\right) \in[0,10]^{2}$ is written as

$$
-c_{i}\left(v_{i}, \theta_{i}\right)+\left(v_{i}-v_{j}\right) \mathbb{1}_{\left\{\alpha^{V C G}\left(v_{B}, v_{S}\right)=a_{i}\right\}}
$$

where $j$ is the other agent. 
Let us analyze the ex ante investment stage. First, it is easy to see that the socially efficient investments $\left(v_{B}^{*}, v_{S}^{*}\right)=(3,0)$ are achieved in equilibrium. Now, suppose that $S$ chooses an ex ante investment $v_{S}=2$. If $B$ chooses $v_{B} \geq 2$ and realizes the baseball project, then the VCG payment for $B$ would be 2 , which exceeds $B$ 's maximum net utility $\frac{3}{2}$. That is,

$$
-\frac{1}{6}\left(v_{B}\right)^{2}+\left(v_{B}-2\right) \mathbb{1}_{\left\{v_{B} \geq 2\right\}} \leq \frac{3}{2}-2<0
$$

for any $v_{B} \in[2,10]$. Thus, $B$ does not have an incentive to achieve $a_{B}$ by investing more than $S$. For $S$, it is clear that choosing 2 is optimal given that $B$ does not invest. Therefore, $\left(v_{B}, v_{S}\right)=(0,2)$ is also an SPE outcome of the entire game, but obviously this is not chosen by efficient $F^{V C G}$.

\section{Discussions}

\subsection{Budget Balance}

In this subsection, we consider an additional requirement for social choice rules: budget balance. This is especially important in the provision of public goods as the cost must be covered by the participants of the mechanism. Budget balance can also be considered as part of efficiency if the transfers collected by the social planner are regarded as a loss of welfare.

Definition 9. A social choice rule $F: \Theta \rightarrow 2^{A \times \mathbb{R}^{I} \times V^{2}} \backslash\{\emptyset\}$ is budget-balanced if for any $\theta \in \Theta$ and $(a, t,(v, \bar{v})) \in F(\theta), \sum_{i \in I} t_{i}=0$ holds. A transfer rule $\tau: U \rightarrow \mathbb{R}^{I}$ is budget-balanced if $\sum_{i \in I} \tau_{i}(u)=0$ holds for any $u \in U$.

Our main finding in this subsection is that budget balance is still compatible with commitment-proofness and efficiency; i.e., there exists $(\alpha, \tau)$ that is commitment-proof, efficient and budget-balanced. This result contrasts with the well-known theorem that there is no social choice function that is strategy-proof, efficient and budget-balanced (Green and Laffont, 1977; Hölmstrom, 1979; Walker, 1980).

Proposition 3. For any efficient allocation rule $\alpha: U \rightarrow A$, there exists a budget-balanced transfer rule $\tau: U \rightarrow \mathbb{R}^{I}$ such that $(\alpha, \tau)$ is commitment-proof.

Proposition 3 is shown by finding a specific transfer rule $\tau$ : for any agent $i \in I, \tau_{i}$ is defined by

$$
\tau_{i}(u)=u_{i}(\alpha(u))-\frac{1}{n} \sum_{i \in I} u_{i}(\alpha(u))
$$


First, it is clear that this $\tau$ is budget-balanced. To see why $(\alpha, \tau)$ is commitment-proof, consider the utility change from $u_{i}(\alpha(u))-\tau_{i}(u)$ to $\tilde{u}_{i}\left(\alpha\left(\tilde{u}_{i}, u_{-i}\right)\right)-\tau_{i}\left(\tilde{u}_{i}, u_{-i}\right)$ when $i$ 's valuation function changes from $u_{i}$ to $\tilde{u}_{i}$. By the structure of $\tau$, it is exactly $\frac{1}{n}$ of the change in the social welfare. This means that the utility change from $u_{i}(\alpha(u))-\tau_{i}(u)$ to $\tilde{u}_{i}\left(\alpha\left(\tilde{u}_{i}, u_{-i}\right)\right)-\tau_{i}\left(\tilde{u}_{i}, u_{-i}\right)$ is always less than zero or $\max _{a \in A}\left\{\tilde{u}_{i}(a)-u_{i}(a)\right\}$, and hence, the inequality of commitmentproofness is satisfied. It is easy to see that this $(\alpha, \tau)$ is not strategy-proof because the agents have an incentive to underreport their valuations to reduce their payment.

By the result of Theorem 1, we obtain the following corollary: budget balance is compatible with the implementation of efficient social choice rules.

Corollary 1. There exists a social choice rule $F: \Theta \rightarrow 2^{A \times \mathbb{R}^{I} \times V^{2}} \backslash\{\emptyset\}$ that is efficient, budget-balanced and implementable in subgame-perfect equilibria for any discount factor $\delta \in$ $(0,1)$.

\subsection{Incomplete Information}

In the main model, we assumed that the agents have complete information about the cost types. In fact, we can show that complete information is not necessary for inducing efficient investment incentives. In incomplete information environments, we would need a tighter condition for implementing efficient allocations and transfers since not all allocation and transfer rules are implementable even with extensive form mechanisms. In this subsection, we provide the following sufficient condition for the implementability of an efficient $F$ under incomplete information: strategy-proofness of the associated $(\alpha, \tau)$.

We consider a model where each agent $i$ knows her own cost type $\theta_{i} \in \Theta_{i}$, but may be unsure about the other agents' cost types $\theta_{-i} \equiv\left(\theta_{j}\right)_{j \in I \backslash\{i\}}$. The agents have a common prior on $\Theta$, denoted by $p$. Conditional on knowing her own cost type $\theta_{i}$, agent $i$ 's posterior distribution over $\Theta_{-i} \equiv \times_{j \in I \backslash\{i\}} \Theta_{j}$ is denoted by $p\left(\cdot \mid \theta_{i}\right)$. For simplicity, we assume that $V^{\theta_{i}}$ and $\Theta_{i}$ are both finite sets in this subsection. ${ }^{19}$ The prior belief is diffuse, i.e., $p(\theta)>0$ for any $\theta \in \Theta$, and $p\left(\cdot \mid \theta_{i}\right)$ is computed by Bayes' rule.

In this section, we consider a reduced version of the entire game: the investment game induced by a pair of allocation and transfer rules $(\alpha, \tau)$. Here, the mechanism stage of the entire game is replaced by functions $(\alpha, \tau)$, and the game simply consists of the ex ante and ex post investment stages. In this Bayesian setting, the investment strategies are defined

\footnotetext{
${ }^{19}$ In one of the extensions, Duggan (1998) argued that the finiteness of these sets is not necessary for implementing Bayesian incentive compatible social choice functions. However, we assume finiteness as it makes the model simpler and does not change the main argument of our result.
} 
in the following way. The set of ex ante investment strategies for agent $i$ is the set of all mappings from $\Theta_{i}$ to $V_{i}$, denoted by $\Sigma_{i}$. The set of ex post investment strategies for agent $i$ is the set of all mappings from $V^{\theta_{i}} \times V_{-i} \times A \times \Theta_{i}$ to $V^{\theta_{i}}$, denoted by $\overline{\mathcal{M}}_{i}$. Let $\Sigma \equiv \times_{i \in I} \Sigma_{i}$ and $\overline{\mathcal{M}} \equiv \times_{i \in I} \overline{\mathcal{M}}_{i}$. First, we define a perfect Bayesian equilibrium of the investment game induced by $(\alpha, \tau)$.

Definition 10. A profile of investment strategies $\left(\sigma^{*}, \mu^{*}\right) \in \Sigma \times \overline{\mathcal{M}}$ is a perfect Bayesian equilibrium (PBE) of the investment game induced by a pair of allocation and transfer rules $(\alpha, \tau)$ given a discount factor $\delta \in(0,1)$ if for each $i \in I$ and $\theta_{i} \in \Theta_{i}$,

1. $\mu_{i}^{*}\left(v, a, \theta_{i}\right) \in \underset{\bar{v}_{i} \in \bar{V}_{i}\left(v_{i}, \theta_{i}\right)}{\arg \max }\left\{\bar{v}_{i}(a)-c_{i}\left(\bar{v}_{i}, \theta_{i}\right)\right\}$ for any $v \in V^{\theta_{i}} \times V_{-i}$ and $a \in A$, and

2. $\sigma_{i}^{*}\left(\theta_{i}\right) \in \underset{v_{i} \in V^{\theta_{i}}}{\arg \max }\left\{-c_{i}\left(v_{i}, \theta_{i}\right)+\delta \sum_{\theta_{-i} \in \Theta_{-i}} p\left(\theta_{-i} \mid \theta_{i}\right)\right.$

$$
\begin{aligned}
& {\left[\mu_{i}^{*}\left(v_{i}, \sigma_{-i}^{*}\left(\theta_{-i}\right), \alpha\left(u^{\theta_{i}, v_{i}}, u_{-i}\right), \theta_{i}\right)\left(\alpha\left(u^{\theta_{i}, v_{i}}, u_{-i}\right)\right)-\tau_{i}\left(u^{\theta_{i}, v_{i}}, u_{-i}\right)\right.} \\
& \left.\left.-c_{i}\left(\mu_{i}^{*}\left(v_{i}, \sigma_{-i}^{*}\left(\theta_{-i}\right), \alpha\left(u^{\theta_{i}, v_{i}}, u_{-i}\right), \theta_{i}\right), \theta_{i}\right)+c_{i}\left(v_{i}, \theta_{i}\right)\right]\right\}
\end{aligned}
$$

where $u_{-i} \equiv u^{\theta_{-i}, \sigma_{-i}^{*}\left(\theta_{-i}\right)}$

hold. Let $\operatorname{PBE}(\theta, \alpha, \tau, \delta) \equiv\left\{(a, t,(v, \bar{v})) \in A \times \mathbb{R}^{I} \times V^{2} \mid \exists \operatorname{PBE}(\sigma, \mu)\right.$ of the investment game induced by $(\alpha, \tau)$ given $\delta$ s.t. $\left(\alpha\left(u^{\theta, \sigma(\theta)}\right), \tau\left(u^{\theta, \sigma(\theta)}\right)\right)=(a, t)$ and $\left.\mu_{i}\left(v, a, \theta_{i}\right)=\bar{v}_{i} \forall i \in I\right\}$ denote the set of all outcomes and valuation functions that are on the equilibrium paths of the investment game given $(\alpha, \tau)$ and $\delta$ when $\theta$ is realized.

Note that in the ex post investment stage, we do not need to specify the beliefs of agents because other agents' cost types are irrelevant to their decisions in this stage.

Now, consider the implementability of $(\alpha, \tau)$. In the literature, several papers have identified the class of social choice functions that can be implemented by extensive form mechanisms in this Bayesian setting (Brusco, 1995; Bergin and Sen, 1998; Duggan, 1998; Baliga, 1999). These studies differ in the generality of the model and the equilibrium concept (PBE or sequential equilibrium), but it is common that they require Bayesian incentive compatibility. Moreover, in the simplest model of Duggan (1998) where quasi-linear utility is employed, he shows that any Bayesian incentive compatible social choice function is implementable in perfect Bayesian equilibria and sequential equilibria. ${ }^{20}$ Based on these arguments, we directly include Bayesian incentive compatibility of $(\alpha, \tau)$ in the definition of the PBE implementability of $F$.

\footnotetext{
${ }^{20}$ Duggan (1998) required value-measurability for implementation, in the sense that chosen outcomes do not change unless at least one agent's preference does, but $(\alpha, \tau)$ in this paper automatically satisfies this.
} 
Our model has an ex ante investment stage, and the belief system at the mechanism stage is endogenously formed by the observations of ex ante investments and the agents' investment strategies. Let $p\left(\theta_{-i} \mid \theta_{i}, v, \sigma\right)$ be agent $i$ 's belief on $\theta_{-i}$ given her own cost type, observed ex ante investments and all agents' investment strategies. This is computed by Bayes' rule:

$$
\begin{aligned}
& p\left(\theta_{-i} \mid \theta_{i}, v, \sigma\right) \\
& \equiv \begin{cases}\frac{p\left(\theta_{-i} \mid \theta_{i}\right) \Pi_{j \in I \backslash\{i\}} \mathbb{1}_{\left\{\sigma_{j}\left(\theta_{j}\right)=v_{j}\right\}}}{\sum_{\tilde{\theta}_{-i} \in \Theta_{-i}} p\left(\tilde{\theta}_{-i} \mid \theta_{i}\right) \Pi_{j \in I \backslash\{i\}} \mathbb{1}_{\left\{\sigma_{j}\left(\tilde{\theta}_{j}\right)=v_{j}\right\}}} & \text { if } \sum_{\tilde{\theta}_{-i} \in \Theta_{-i}} p\left(\tilde{\theta}_{-i} \mid \theta_{i}\right) \Pi_{j \in I \backslash\{i\}} \mathbb{1}_{\left\{\sigma_{j}\left(\tilde{\theta}_{j}\right)=v_{j}\right\}}>0, \\
\text { any probability distribution over } \Theta_{-i} & \text { otherwise. }\end{cases}
\end{aligned}
$$

Bayesian incentive compatibility of a social choice function is defined for each information set at the mechanism stage.

Definition 11. A pair of allocation and transfer rules $(\alpha, \tau): U \rightarrow A \times \mathbb{R}^{I}$ is Bayesian incentive compatible at $\theta \in \Theta$ and $v \in V^{\theta}$ given $\sigma \in \Sigma$ if for any $i \in I, \tilde{\theta}_{i} \in \Theta_{i}$ and $\tilde{v}_{i} \in V^{\tilde{\theta}_{i}}$,

$$
\begin{aligned}
& \sum_{\theta_{-i} \in \Theta_{-i}} p\left(\theta_{-i} \mid \theta_{i}, v, \sigma\right)\left[u^{\theta_{i}, v_{i}}\left(\alpha\left(u^{\theta, v}\right)\right)-\tau_{i}\left(u^{\theta, v}\right)\right] \\
\geq & \sum_{\theta_{-i} \in \Theta_{-i}} p\left(\theta_{-i} \mid \theta_{i}, v, \sigma\right)\left[u^{\theta_{i}, v_{i}}\left(\alpha\left(u^{\tilde{\theta}_{i}, \tilde{v}_{i}}, u^{\theta_{-i}, v_{-i}}\right)\right)-\tau_{i}\left(u^{\tilde{\theta}_{i}, \tilde{v}_{i}}, u^{\theta_{-i}, v_{-i}}\right)\right] .
\end{aligned}
$$

Because the planner does not observe the ex ante investments $v,(\alpha, \tau)$ must satisfy Bayesian incentive compatibility for any possible beliefs at the mechanism stage. ${ }^{21}$ The implementability of $F$ with the associated $(\alpha, \tau)$ is defined by the following two conditions: (i) $(\alpha, \tau)$ must be Bayesian incentive compatible given the PBE investment strategy in every information set at the mechanism stage, and (ii) the set of PBE outcomes of the investment game coincides with $F$ for any $\theta \in \Theta$.

Definition 12. Given a discount factor $\delta \in(0,1)$, social choice rule $F: \Theta \rightarrow 2^{A \times \mathbb{R}^{I} \times V^{2}} \backslash\{\emptyset\}$ with the associated allocation and transfer rules $(\alpha, \tau): U \rightarrow A \times \mathbb{R}^{I}$ is implementable in perfect Bayesian equilibrium if

1. $(\alpha, \tau)$ is Bayesian incentive compatible at any $\theta \in \Theta$ and $v \in V^{\theta}$ given any $\sigma^{*} \in \Sigma$ such that $\sigma^{*}$ is part of a PBE, and

2. $F(\theta)=P B E(\theta, \alpha, \tau, \delta)$ for any $\theta \in \Theta$.

\footnotetext{
${ }^{21}$ Duggan (1998) also considered global implementation, in which the social planner elicits information regarding agents' belief systems and implements different social choice functions depending on their belief systems. However, we do not consider this in our paper and leave it for future research.
} 
Proposition 4 is our main result in this incomplete information setting.

Proposition 4. Consider an efficient social choice rule $F: \Theta \rightarrow 2^{A \times \mathbb{R}^{I} \times V^{2}} \backslash\{\emptyset\}$ and a pair of allocation and transfer rules $(\alpha, \tau): U \rightarrow A \times \mathbb{R}^{I}$ that is associated with $F$. $F$ is implementable in perfect Bayesian equilibria for any discount factor $\delta \in(0,1)$ if $(\alpha, \tau)$ is strategy-proof.

The key idea is that even under incomplete information, the least costly investments are chosen in any equilibrium of the investment game as long as the associated $(\alpha, \tau)$ is commitment-proof. We provide a stronger sufficient condition (strategy-proofness) than commitment-proofness just to satisfy Bayesian incentive compatibility for any beliefs of agents at the mechanism stage.

\section{Concluding Remarks}

The main contribution of our paper is to provide a sufficient and necessary condition for an efficient social choice rule to be implementable when there are endogenous investments. Moreover, the commitment-proofness condition is defined for $(\alpha, \tau)$, which is interpreted as a standard social choice function where investments are exogenously given. Therefore, when the assumptions regarding the investment environment are satisfied, the social planner can simply design a mechanism so that $(\alpha, \tau)$ satisfies commitment-proofness. Commitment-proofness is not a strong requirement because Proposition 3 shows the existence of a commitment-proof, efficient and budget-balanced social choice function.

In this paper, the assumptions regarding the technology of investments are somewhat strong. In particular, (i) the investment technology has no uncertainty, and (ii) ex post investment is available and is strictly less costly than the ex ante one. ${ }^{22}$ These assumptions allow us to easily characterize efficient investments, and the goal was to eliminate incentives for any ex ante investments. In future research, these assumptions can be relaxed in the following general ways. Agents may make uncertain ex ante investments, which would introduce a new source of uncertainty to the model. Moreover, some positive ex ante investment could be socially efficient while ex post investments are still available. These extensions would more closely connect our paper to Piccione and Tan (1996) and other papers on information acquisition (Bergemann and Välimäki, 2002; Obara, 2008). Under these more general settings, we hope to obtain conditions for which efficient social choice rules are implementable.

\footnotetext{
${ }^{22}$ Although our result still holds for some systematic changes in the cost functions after the mechanism is run (see footnote 12), we do not know what will happen if the ex post cost function is uncertain ex ante.
} 


\section{References}

Aghion, P., M. Dewatripont, and P. Rey (1994): "Renegotiation Design with Unverifiable Information," Econometrica, 62, 257-282.

Aghion, P., D. Fudenberg, R. Holden, T. Kunimoto, and O. Tercieux (2012): "Subgame-Perfect Implementation Under Information Perturbations," The Quarterly Journal of Economics, 127, 1843-1881.

Arozamena, L. and E. Cantillon (2004): "Investment Incentives in Procurement Auctions," Review of Economic Studies, 71, 1-18.

BAG, P. K. (1997): "Optimal Auction Design and R\&D," European Economic Review, 41, $1655-1674$.

Baliga, S. (1999): "Implementation in Economic Environments with Incomplete Information: The Use of Multi-Stage Games," Games and Economic Behavior, 27, 173-183.

Bergemann, D. And J. VÄLImÄKi (2002): "Information Acquisition and Efficient Mechanism Design," Econometrica, 70, 1007-1033.

Bergin, J. And A. Sen (1998): "Extensive Form Implementation in Incomplete Information Environments," Journal of Economic Theory, 80, 222-256.

Brusco, S. (1995): "Perfect Bayesian Implementation," Economic Theory, 5, 419-444.

Chen, Y.-C., R. Holden, T. Kunimoto, Y. Sun, and T. Wilkening (2018): "Getting Dynamic Implementation to Work," Unpublished manuscript.

Cole, H. L., G. J. Mailath, And A. Postlewaite (2001a): "Efficient Non-Contractible Investments in Finite Economies," Advances in Theoretical Economics, 1, Article 2.

(2001b): "Efficient Non-Contractible Investments in Large Economies," Journal of Economic Theory, 101, 333-373.

De Meza, D. And B. Lockwood (2010): "Too Much Investment? A Problem of Endogenous Outside Options," Games and Economic Behavior, 69, 503-511.

Duggan, J. (1998): "An Extensive Form Solution to the Adverse Selection Problem in Principal/Multi-agent Environments," Review of Economic Design, 3, 167-191. 
Felli, L. And K. Roberts (2002): "Does Competition Solve the Hold-up Problem?" CEPR Discussion Paper.

Green, J. And J.-J. Laffont (1977): "Characterization of Satisfactory Mechanisms for the Revelation of Preferences for Public Goods," Econometrica, 45, 427-438.

Grossman, S. And O. Hart (1986): "The Costs and Benefits of Ownership: A Theory of Vertical and Lateral Integration," Journal of Political Economy, 94, 691-719.

Gul, F. (2001): "Unobservable Investment and the Hold-up Problem," Econometrica, 69, $343-376$.

Hart, O. (1995): Firms, Contracts, and Financial Structure, Oxford University Press.

Hart, O. And J. Moore (1988): "Incomplete Contracts and Renegotiation," Econometrica, 56, 755-785.

- (1990): "Property rights and the nature of the firm," Journal of Political Economy, 98, 1119-1158.

Hatfield, J. W., F. Kojima, and S. D. Kominers (2018): "Strategy-Proofness, Investment Efficiency, and Marginal Returns: An Equivalence," SSRN Working Paper.

Hölmstrom, B. (1979): "Groves' Scheme on Restricted Domains," Econometrica, 47, 11371144.

Klein, B., R. G. Crawford, and A. A. Alchian (1978): "Vertical Integration, Appropriable Rents, and the Competitive Contracting Process," Journal of Law and Economics, 21, 297-326.

Laffont, J.-J. And J. Tirole (1986): "Using Cost Observation to Regulate Firms," Journal of Political Economy, 94, 614-641.

_- (1987): "Auctioning Incentive Contracts," Journal of Political Economy, 95, 921-937.

Loertscher, S. And M. H. Riordan (2019): "Make and Buy: Outsourcing, Vertical Integration, and Cost Reduction," American Economic Journal: Microeconomics, 11, 105123.

Mailath, G. J., A. Postlewaite, and L. Samuelson (2013): "Pricing and Investments in Matching Markets," Theoretical Economics, 8, 535-590. 
Maskin, E. AND J. Tirole (1999): "Unforeseen Contingencies and Incomplete Contracts," Review of Economic Studies, 66, 83-114.

Matsushima, H. And S. Noda (2016): "Mechanism Design in Hidden Action and Hidden Information: Richness and Pure Groves," CARF Working Paper.

McAfee, R. P. And J. McMillan (1986): "Bidding for Contracts: A Principal-Agent Analysis," Rand Journal of Economics, 17, 326-338.

Milgrom, P. (1981): "Rational Expectations, Information Acquisition, and Competitive Bidding," Econometrica, 49, 921-943.

Moore, J. AND R. Repullo (1988): "Subgame Perfect Implementation," Econometrica, $56,1191-1220$.

Nöldeke, G. AND L. Samuelson (2015): "Investment and Competitive Matching," Econometrica, 83, 835-896.

ObarA, I. (2008): "The Full Surplus Extraction Theorem with Hidden Actions," BE Journal of Theoretical Economics, 8, 1-28.

Piccione, M. And G. TAn (1996): "Cost-Reducing Investment, Optimal Procurement and Implementation by Auctions," International Economic Review, 37, 663-685.

Rogerson, W. P. (1992): "Contractual Solutions to the Hold-Up Problems," Review of Economic Studies, 59, 777-793.

Stegeman, M. (1996): "Participation Costs and Efficient Auctions," Journal of Economic Theory, 71, 228-259.

TAN, G. (1992): "Entry and R\&D in Procurement Contracting," Journal of Economic Theory, 58, 41-60.

Tomoeda, K. (2017): "First-price Auction Implements Efficient Investments," Economics Letters, 159, 198-200.

Walker, M. (1980): "On the Nonexistence of a Dominant Strategy Mechanism for Making Optimal Public Decisions," Econometrica, 48, 1521-1540.

Williamson, O. E. (1979): "Transaction Cost Economics: The Governance of Contractual Relations," Journal of Law and Economics, 22, 233-261. 
- (1983): "Credible Commitments: Using Hostages to Support Exchange," American Economic Review, 73, 519-540. 


\section{Appendix A Proofs of the Main Results}

\section{Appendix A.1 Proof of Proposition 1}

To show that the associated $(\alpha, \tau)$ is uniquely determined by $F$, it suffices to show that for every $u \in U$, there exist $\theta \in \Theta$ and $(a, t,(v, \bar{v})) \in F(\theta)$ such that $u^{\theta, v}=u$.

By the efficiency of $F$ and $\delta \in(0,1)$, the efficient choice of ex ante investment should always be $v$ with the lowest cost; i.e., for any $\theta \in \Theta$ and $(a, t,(v, \bar{v})) \in F(\theta)$, we have $v \in Z^{\theta}$. Now, take any $u \in U$. By definition, there exist $\theta \in \Theta$ and $v \in V^{\theta}$ such that $u^{\theta, v}=u$. By the richness of $\Theta$, we can find $\tilde{\theta} \in \Theta$ such that

- $c_{i}\left(v_{i}, \tilde{\theta}_{i}\right)=0$,

- $V_{i}^{\tilde{\theta}_{i}}=\bar{V}_{i}\left(v_{i}, \theta_{i}\right)$, and

- $c_{i}\left(\bar{v}_{i}, \tilde{\theta}_{i}\right)=c_{i}\left(\bar{v}_{i}, \theta_{i}\right)-c_{i}\left(v_{i}, \theta_{i}\right)$ for any $\bar{v}_{i} \in \bar{V}_{i}\left(v_{i}, \theta_{i}\right)$

for all $i \in I$. Then, we can show that $u^{\tilde{\theta}, \tilde{v}}=u$ holds for any $\tilde{v} \in Z^{\tilde{\theta}}$ : for any $i \in I$ and $a \in A$,

$$
\begin{aligned}
u_{i}(a) & =u_{i}^{\theta_{i}, v_{i}}(a) \\
& =\max _{\bar{v}_{i} \in \bar{V}_{i}\left(v_{i}, \theta_{i}\right)}\left\{\bar{v}_{i}(a)-c_{i}\left(\bar{v}_{i}, \theta_{i}\right)\right\}+c_{i}\left(v_{i}, \theta_{i}\right) \\
& =\max _{\bar{v}_{i} \in V_{i}}\left\{\bar{v}_{i}(a)-c_{i}\left(\bar{v}_{i}, \tilde{\theta}_{i}\right)\right\} \\
& =u_{i}^{\tilde{\theta}_{i}, \tilde{v}_{i}}(a) .
\end{aligned}
$$

This implies that there exists $\left(\tilde{a}, \tilde{t},\left(\tilde{v}, \tilde{v}^{\prime}\right)\right) \in F(\tilde{\theta})$ with $u^{\tilde{\theta}, \tilde{v}}=u$, and $(\alpha(u), \tau(u))$ is uniquely determined to be $(\tilde{a}, \tilde{t})$ for any $(\alpha, \tau)$ that is associated with $F$.

Moreover, by the efficiency of $F$ and $\tilde{v} \in Z^{\tilde{\theta}}, \alpha(u)$ must satisfy

$$
\alpha(u)=\tilde{a} \in \underset{a \in A}{\arg \max } \sum_{i \in I}\left\{-c_{i}\left(\tilde{v}_{i}, \tilde{\theta}_{i}\right)+\delta u^{\tilde{\theta}_{i}, \tilde{v}_{i}}(a)\right\}=\underset{a \in A}{\arg \max } \sum_{i \in I} u^{\tilde{\theta}_{i}, \tilde{v}_{i}}(a),
$$

which requires that $\alpha$ be efficient.

\section{Appendix A.2 Proof of Theorem 1}

[1] If part.

Take any discount factor $\delta \in(0,1)$ and fix it.

First, consider the ex post investment stage. Since this stage is a single-agent optimization problem and it does not involve a mechanism, in any subgame starting from the terminal 
node $n(\theta, v) \in T(\theta, v)$ of the mechanism for any $(\theta, v)$, the equilibrium investment choice of every agent is socially efficient.

Next, consider the mechanism stage starting from each initial node $n_{0}(\theta, v)$ of the mechanism for each $(\theta, v)$. Here, since the pair of associated allocation and transfer rules $(\alpha, \tau)$ : $U \rightarrow A \times \mathbb{R}^{I}$ is unique, consider a Moore-Repullo mechanism $m$ that implements $(\alpha, \tau)$ in SPE in the mechanism stage of each subgame. That is, the SPE outcome (alternative and transfers) of $m$ is $\left(\alpha\left(u^{\theta, v}\right), \tau\left(u^{\theta, v}\right)\right)$ in any subgame starting from the initial node $n_{0}(\theta, v)$ of the mechanism. Then, since $(\alpha, \tau)$ is associated with $F$, mechanism $m$ implements $(a, t)$ that is consistent with $F$ for any given $(\theta, v)$.

The main part of the proof is to show that when $(\alpha, \tau)$ is commitment-proof, efficient $e x$ ante investments specified by $F$ are chosen in the SPE of the entire game induced by this mechanism $m$. According to the definition of efficiency, it is clear that the efficient ex ante investment should always be $v$ with the lowest cost; i.e., for any $\theta \in \Theta$ and $(a, t,(v, \bar{v})) \in$ $F(\theta)$, we have $v \in Z^{\theta}$. Therefore, it suffices to show that for any $\theta \in \Theta$, the equilibrium $e x$ ante investments are characterized by $Z^{\theta}$.

Take any $\theta \in \Theta$, any $i \in I$ and $v_{-i} \in V^{\theta_{-i}}$ and consider $i$ 's incentive for ex ante investments when the valuation functions of other agents at the mechanism stage are fixed to $u_{-i} \equiv u^{\theta_{-i}, v_{-i}}$. Consider two different choices: any $v_{i}^{0} \in Z^{\theta_{i}}$ and any $v_{i} \in V^{\theta_{i}} \backslash Z^{\theta_{i}}$. We can show that $v_{i}^{0}$ gives a strictly higher utility than $v_{i}$ for $i$. To see this, let $\mu_{i}^{*}: V^{\theta} \rightarrow V^{\theta_{i}}$ be the optimal ex post investment function that selects the optimal ex post investment $\bar{v}_{i}$ for each ex ante investments $v \in V$ given $(\alpha, \tau)$. The ex ante utility from $v_{i}$ given $v_{-i}$ is written as:

$$
\begin{aligned}
& -c_{i}\left(v_{i}, \theta_{i}\right)+\delta\left[\mu_{i}^{*}(v)\left(\alpha\left(u^{\theta_{i}, v_{i}}, u_{-i}\right)\right)-\tau_{i}\left(u^{\theta_{i}, v_{i}}, u_{-i}\right)-\left(c_{i}\left(\mu_{i}^{*}(v), \theta_{i}\right)-c_{i}\left(v_{i}, \theta_{i}\right)\right)\right] \\
= & \delta\left[\mu_{i}^{*}(v)\left(\alpha\left(u^{\theta_{i}, v_{i}}, u_{-i}\right)\right)-\tau_{i}\left(u^{\theta_{i}, v_{i}}, u_{-i}\right)-c_{i}\left(\mu_{i}^{*}(v), \theta_{i}\right)\right]-(1-\delta) c_{i}\left(v_{i}, \theta_{i}\right) \\
< & \delta\left[\mu_{i}^{*}(v)\left(\alpha\left(u^{\theta_{i}, v_{i}}, u_{-i}\right)\right)-\tau_{i}\left(u^{\theta_{i}, v_{i}}, u_{-i}\right)-c_{i}\left(\mu_{i}^{*}(v), \theta_{i}\right)\right] \\
= & \delta\left[u^{\theta_{i}, v_{i}}\left(\alpha\left(u^{\theta_{i}, v_{i}}, u_{-i}\right)\right)-\tau_{i}\left(u^{\theta_{i}, v_{i}}, u_{-i}\right)-c_{i}\left(v_{i}, \theta_{i}\right)\right] \\
\leq & \delta\left[u^{\theta_{i}, v_{i}}\left(\alpha\left(u^{\theta_{i}, v_{i}}, u_{-i}\right)\right)-\tau_{i}\left(u^{\theta_{i}, v_{i}}, u_{-i}\right)-\max \left\{0, \max _{a \in A}\left\{u^{\theta_{i}, v_{i}}(a)-u^{\theta_{i}, v_{i}^{0}}(a)\right\}\right\}\right] \\
\leq & \delta\left[u^{\theta_{i}, v_{i}^{0}}\left(\alpha\left(u^{\theta_{i}, v_{i}^{0}}, u_{-i}\right)\right)-\tau_{i}\left(u^{\theta_{i}, v_{i}^{0}}, u_{-i}\right)\right] \\
= & \delta\left[\mu_{i}^{*}\left(v_{i}^{0}, v_{-i}\right)\left(\alpha\left(u^{\theta_{i}, v_{i}^{0}}, u_{-i}\right)\right)-\tau_{i}\left(u^{\theta_{i}, v_{i}^{0}}, u_{-i}\right)-c_{i}\left(\mu_{i}^{*}\left(v_{i}^{0}, v_{-i}\right), \theta_{i}\right)\right],
\end{aligned}
$$

in which the last equation (9) is the ex ante utility from choosing $v_{i}^{0}$ given $v_{-i}$. The inequality in (5) holds because $c_{i}\left(v_{i}, \theta_{i}\right)>0$ and $\delta<1$; the equality in (6) follows from the definition of $u^{\theta_{i}, v_{i}}$; the inequality in (7) follows from Lemma 1; the inequality in (8) follows from the 
fact that $(\alpha, \tau)$ is commitment-proof; and the equality in (9) follows from the definition of $u^{\theta_{i}, v_{i}^{0}}$. Moreover, consider when $Z^{\theta_{i}}$ is not a singleton set. Since any valuation functions in $Z^{\theta_{i}}$ give exactly the same utility from the calculation above, $Z^{\theta_{i}}$ is the set of best responses for $i$ in the first investment stage to any $v_{-i}$.

From the same argument, for any ex ante investment $v_{j} \in Z^{\theta_{j}}, j$ should have the same valuation at the mechanism stage. Since the utility $i$ obtains from choosing any investment in $Z^{\theta_{i}}$ is unchanged as long as $v_{j}$ is taken from $Z^{\theta_{j}}$ for any $j \in I \backslash\{i\}$, the equilibrium ex ante investments are characterized by $Z^{\theta}$. Thus, we conclude that $F$ is implemented by the mechanism $m$ in SPE for any discount factor $\delta \in(0,1)$.

[2] Only if part.

By Proposition 1, we know that the pair of associated $(\alpha, \tau)$ is unique for efficient $F$. Suppose, for the sake of contradiction, that this $(\alpha, \tau)$ is not commitment-proof. Then, there must be $i \in I, u \in U$ and $\tilde{u}_{i} \in U_{i}$ such that

$$
\tilde{u}_{i}\left(\alpha\left(\tilde{u}_{i}, u_{-i}\right)\right)-\tau_{i}\left(\tilde{u}_{i}, u_{-i}\right)-\left(u_{i}(\alpha(u))-\tau_{i}(u)\right)>\max \left\{0, \max _{a \in A}\left\{\tilde{u}_{i}(a)-u_{i}(a)\right\}\right\} .
$$

Consider the following profile of cost types $\theta$ such that

$$
\begin{aligned}
& V^{\theta_{i}}=\left\{u_{i}, \tilde{u}_{i}\right\}, \\
& V^{\theta_{j}}=\left\{u_{j}\right\}, \text { for all } j \in I \backslash\{i\}, \\
& c_{i}\left(u_{i}, \theta_{i}\right)=0, \\
& c_{i}\left(\tilde{u}_{i}, \theta_{i}\right)= \begin{cases}\max _{a \in A}\left\{\tilde{u}_{i}(a)-u_{i}(a)\right\} & \text { if } \max _{a \in A}\left\{\tilde{u}_{i}(a)-u_{i}(a)\right\}>0, \\
\Delta & \text { otherwise, }\end{cases} \\
& c_{j}\left(u_{j}, \theta_{j}\right)=0 \text { for all } j \in I \backslash\{i\}
\end{aligned}
$$

where $\Delta>0$. The richness condition ensures $\theta \in \Theta$. Note that for this profile of cost types $\theta, F$ specifies $u$ as ex ante investments because $Z^{\theta}=\{u\}$.

Consider any mechanism $m$ that implements $F$ in SPE and the entire game induced by $m$. First, we can see that $U^{\theta_{i}}=\left\{u_{i}, \tilde{u}_{i}\right\}$. Consider the optimal ex post investment in each terminal node of the mechanism. When $i$ chooses $\tilde{u}_{i}$ ex ante, since $c_{i}\left(\tilde{u}_{i}, \theta_{i}\right)>c_{i}\left(u_{i}, \theta_{i}\right)$, the only ex post choice is $\tilde{u}_{i}$ for any $a \in A$. Thus, the valuation at the mechanism stage is also $\tilde{u}_{i}$. On the other hand, when $i$ chooses $u_{i}$ ex ante, she can still choose from $\left\{u_{i}, \tilde{u}_{i}\right\}$ in the ex post stage. However, by the construction of the cost function, we have

$$
u_{i}(a) \geq \tilde{u}_{i}(a)-c_{i}\left(\tilde{u}_{i}, \theta_{i}\right)
$$


for any $a \in A$. Thus, the valuation at the mechanism stage is

$$
u^{\theta_{i}, u_{i}}(a)=\max _{\bar{v}^{i} \in\left\{u_{i}, \tilde{u}_{i}\right\}}\left\{\bar{v}^{i}(a)-c_{i}\left(\bar{v}^{i}, \theta_{i}\right)\right\}=u_{i}(a)
$$

for each $a \in A$. To summarize, $i$ 's optimal investment strategy and the valuation at the mechanism stage are:

\begin{tabular}{c|c|c} 
Ex Ante Valuation & Valuation at the Mechanism & Optimal Ex Post Valuation \\
\hline \hline$u_{i}$ & $u_{i}$ & for any $a: u_{i}\left(\right.$ or $\tilde{u}_{i}$ if $\left.u_{i}(a)=\tilde{u}_{i}(a)-c_{i}\left(\tilde{u}_{i}\right)\right)$ \\
\hline$\tilde{u}_{i}$ & $\tilde{u}_{i}$ & for any $a: \tilde{u}_{i}$
\end{tabular}

Now, consider $i$ 's incentive in the ex ante investment stage: $i$ chooses either $u_{i}$ or $\tilde{u}_{i}$ given other agents' valuations $u_{-i}$. Since $(\alpha, \tau)$ is associated with $F$, we can write the ex ante utility using $(\alpha, \tau): i$ 's ex ante utility when choosing $\tilde{u}_{i}$ is $-c_{i}\left(\tilde{u}_{i}, \theta_{i}\right)+\delta\left[\tilde{u}_{i}\left(\alpha\left(\tilde{u}_{i}, u_{-i}\right)\right)-\tau_{i}\left(\tilde{u}_{i}, u_{-i}\right)\right]$ and that from choosing $u_{i}$ is $\delta\left[u_{i}(\alpha(u))-\tau_{i}(u)\right]$. The difference between these two is

$$
\begin{aligned}
& -c_{i}\left(\tilde{u}_{i}, \theta_{i}\right)+\delta\left[\tilde{u}_{i}\left(\alpha\left(\tilde{u}_{i}, u_{-i}\right)\right)-\tau_{i}\left(\tilde{u}_{i}, u_{-i}\right)\right]-\delta\left[u_{i}(\alpha(u))-\tau_{i}(u)\right] \\
= & -(1-\delta) c_{i}\left(\tilde{u}_{i}, \theta_{i}\right)+\delta\left[\tilde{u}_{i}\left(\alpha\left(\tilde{u}_{i}, u_{-i}\right)\right)-\tau_{i}\left(\tilde{u}_{i}, u_{-i}\right)-c_{i}\left(\tilde{u}_{i}, \theta_{i}\right)\right]-\delta\left[u_{i}(\alpha(u))-\tau_{i}(u)\right] \\
= & -(1-\delta) c_{i}\left(\tilde{u}_{i}, \theta_{i}\right) \\
& +\delta\left[\tilde{u}_{i}\left(\alpha\left(\tilde{u}_{i}, u_{-i}\right)\right)-\tau_{i}\left(\tilde{u}_{i}, u_{-i}\right)-\left(u_{i}(\alpha(u))-\tau_{i}(u)\right)-\max \left\{\Delta, \max _{a \in A}\left\{\tilde{u}_{i}(a)-u_{i}(a)\right\}\right\}\right] \\
> & 0,
\end{aligned}
$$

in which $c_{i}\left(\tilde{u}_{i}, \theta_{i}\right)=\max \left\{\Delta, \max _{a \in A}\left\{\tilde{u}_{i}(a)-u_{i}(a)\right\}\right\}$ holds for sufficiently small $\Delta>0$, and the final inequality holds from equation (10) when we take $\delta$ sufficiently close to 1 and $\Delta>0$ sufficiently small. Therefore, $\tilde{u}_{i}$ is chosen in the first investment stage in any SPE of the entire game induced by $m$, and this contradicts the fact that $m$ implements $F$ in SPE.

\section{A.3 Proof of Proposition 2}

Suppose that there exists a social choice rule $G: \Theta \rightarrow 2^{V \times A \times \mathbb{R}^{I}} \backslash\{\emptyset\}$ that is efficient and implemented by some mechanism $m$ in SPE without ex post investments, and we derive a contradiction.

First, we can see that the pair of associated allocation and transfer rules $(\alpha, \tau): V \rightarrow$ $A \times \mathbb{R}^{I}$ is unique, and the mechanism $m$ implements it in the mechanism stage. This occurs because for any $v \in V,(\alpha(v), \tau(v))$ is uniquely determined as $(a, t)$ that satisfies $(a, t, v) \in G(\theta)$ for $\theta \in \Theta$ with $V^{\theta}=\{v\}$. Moreover, this unique $\alpha$ has to be efficient because for each $v \in V, \alpha(v) \in \underset{a \in A}{\arg \max } \sum_{i \in I} v_{i}(a)$ holds for the cost types $\theta$ with $V^{\theta}=\{v\}$. 
Next, we examine two cases where $(\alpha, \tau)$ is not strategy-proof and $(\alpha, \tau)$ is strategy-proof. Here, strategy-proofness of $(\alpha, \tau)$ is defined in the standard way: for any $i \in I, v \in V$ and $\tilde{v}_{i} \in V_{i}, v_{i}\left(\alpha\left(\tilde{v}_{i}, v_{-i}\right)\right)-\tau_{i}\left(\tilde{v}_{i}, v_{-i}\right) \leq v_{i}(\alpha(v))-\tau_{i}(v)$. In either case, we can find an inefficient investment equilibrium in the entire game without ex post investments induced by $m$, which is a contradiction.

[1] When $(\alpha, \tau)$ is not strategy-proof.

In this case, there are $i \in I, v \in V$ and $\tilde{v}_{i} \in V_{i}$ such that

$$
v_{i}\left(\alpha\left(\tilde{v}_{i}, v_{-i}\right)\right)-\tau_{i}\left(\tilde{v}_{i}, v_{-i}\right)>v_{i}(\alpha(v))-\tau_{i}(v) .
$$

By the richness condition of $\Theta$, we can find cost types $\theta \in \Theta$ such that

$$
\begin{aligned}
& V^{\theta_{i}}=\left\{v_{i}, \tilde{v}_{i}\right\}, \\
& V^{\theta_{j}}=\left\{v_{j}\right\} \text { for all } j \in I \backslash\{i\}, \\
& c_{i}\left(v_{i}, \theta_{i}\right)=\max \left\{0, v_{i}(\alpha(v))-\tau_{i}(v)-\left(\tilde{v}_{i}\left(\alpha\left(\tilde{v}_{i}, v_{-i}\right)\right)-\tau_{i}\left(\tilde{v}_{i}, v_{-i}\right)\right)\right\}, \\
& c_{i}\left(\tilde{v}_{i}, \theta_{i}\right)=\max \left\{0, \tilde{v}_{i}\left(\alpha\left(\tilde{v}_{i}, v_{-i}\right)\right)-\tau_{i}\left(\tilde{v}_{i}, v_{-i}\right)-\left(v_{i}(\alpha(v))-\tau_{i}(v)\right)\right\}, \text { and } \\
& c_{j}\left(v_{j}, \theta_{j}\right)=0 \text { for all } j \in I \backslash\{i\} .
\end{aligned}
$$

First, consider any mechanism $m$ that implements $(\alpha, \tau)$ in the mechanism stage, and consider $i$ 's incentive in the ex ante investment stage. The ex ante utility from choosing $v_{i}$ is $-c_{i}\left(v_{i}, \theta_{i}\right)+v_{i}(\alpha(v))-\tau_{i}(v)$, and that from choosing $\tilde{v}_{i}$ is $-c_{i}\left(\tilde{v}_{i}, \theta_{i}\right)+\tilde{v}_{i}\left(\alpha\left(\tilde{v}_{i}, v_{-i}\right)\right)-\tau_{i}\left(\tilde{v}_{i}, v_{-i}\right)$. The difference is

$$
\begin{aligned}
& -c_{i}\left(v_{i}, \theta_{i}\right)+v_{i}(\alpha(v))-\tau_{i}(v)-\left\{-c_{i}\left(\tilde{v}_{i}, \theta_{i}\right)+\tilde{v}_{i}\left(\alpha\left(\tilde{v}_{i}, v_{-i}\right)\right)-\tau_{i}\left(\tilde{v}_{i}, v_{-i}\right)\right\} \\
= & v_{i}(\alpha(v))-\tau_{i}(v)-\left(\tilde{v}_{i}\left(\alpha\left(\tilde{v}_{i}, v_{-i}\right)\right)-\tau_{i}\left(\tilde{v}_{i}, v_{-i}\right)\right)-\left(c_{i}\left(v_{i}, \theta_{i}\right)-c_{i}\left(\tilde{v}_{i}, \theta_{i}\right)\right) \\
= & 0 .
\end{aligned}
$$

Therefore, $v_{i}$ and $\tilde{v}_{i}$ are indifferent for $i$, and both $(\alpha(v), \tau(v), v) \in S P E(\theta, m)$ and $\left(\alpha(v), \tau(v),\left(\tilde{v}_{i}, v_{-i}\right)\right) \in$ $\operatorname{SPE}(\theta, m)$ hold.

Next, consider whether $v$ and $\left(\tilde{v}_{i}, v_{-i}\right)$ are chosen by $G$. For $v$, the social welfare is

$$
\sum_{j \in I}\left\{-c_{j}\left(v_{j}, \theta_{j}\right)+v_{j}(\alpha(v))\right\}=-c_{i}\left(v_{i}, \theta_{i}\right)+\sum_{j \in I} v_{j}(\alpha(v)) .
$$

And for $\left(\tilde{v}_{i}, v_{-i}\right)$, the social welfare is

$$
-c_{i}\left(\tilde{v}_{i}, \theta_{i}\right)+\tilde{v}_{i}\left(\alpha\left(\tilde{v}_{i}, v_{-i}\right)\right)+\sum_{j \in I \backslash\{i\}} v_{j}\left(\alpha\left(\tilde{v}_{i}, v_{-i}\right)\right) .
$$


The difference between these two is:

$$
\begin{aligned}
& -c_{i}\left(v_{i}, \theta_{i}\right)+\sum_{j \in I} v_{j}(\alpha(v))-\left\{-c_{i}\left(\tilde{v}_{i}, \theta_{i}\right)+\tilde{v}_{i}\left(\alpha\left(\tilde{v}_{i}, v_{-i}\right)\right)+\sum_{j \in I \backslash\{i\}} v_{j}\left(\alpha\left(\tilde{v}_{i}, v_{-i}\right)\right)\right\}(12) \\
\geq & \sum_{j \in I} v_{j}\left(\alpha\left(\tilde{v}_{i}, v_{-i}\right)\right)-\tilde{v}_{i}\left(\alpha\left(\tilde{v}_{i}, v_{-i}\right)\right)-\sum_{j \in I \backslash\{i\}} v_{j}\left(\alpha\left(\tilde{v}_{i}, v_{-i}\right)\right)-\left(c_{i}\left(v_{i}, \theta_{i}\right)-c_{i}\left(\tilde{v}_{i}, \theta_{i}\right)\right) \\
= & v_{i}\left(\alpha\left(\tilde{v}_{i}, v_{-i}\right)\right)-\tilde{v}_{i}\left(\alpha\left(\tilde{v}_{i}, v_{-i}\right)\right)-\left(c_{i}\left(v_{i}, \theta_{i}\right)-c_{i}\left(\tilde{v}_{i}, \theta_{i}\right)\right) \\
> & v_{i}(\alpha(v))-\tau_{i}(v)+\tau_{i}\left(\tilde{v}_{i}, v_{-i}\right)-\tilde{v}_{i}\left(\alpha\left(\tilde{v}_{i}, v_{-i}\right)\right)-\left(c_{i}\left(v_{i}, \theta_{i}\right)-c_{i}\left(\tilde{v}_{i}, \theta_{i}\right)\right) \\
= & 0
\end{aligned}
$$

in which the inequality in (13) follows from the efficiency of $\alpha$; and the inequality in (15) follows from equation (11). Therefore, we have $(\alpha(v), \tau(v), v) \in G(\theta, m)$, but $\left(\alpha(v), \tau(v),\left(\tilde{v}_{i}, v_{-i}\right)\right) \notin$ $G(\theta, m)$. This contradicts $G(\theta)=\operatorname{SPE}(\theta, m)$.

$[2]$ When $(\alpha, \tau)$ is strategy-proof.

We embed the example in Section 3 into any problem. ${ }^{23}$ Consider any $(\alpha, \tau)$ that is efficient and strategy-proof. Suppose $\{i, j\} \subseteq I$ and $\left\{a_{i}, a_{j}\right\} \subseteq A$. By the assumption of $V$ and $\Theta$, we can find cost types $\theta \in \Theta$ such that

$$
\begin{aligned}
& V^{\theta_{i}}=\left\{x \mathbb{1}_{\left\{a=a_{i}\right\}}: x \in[0, \kappa]\right\}, \\
& V^{\theta_{j}}=\left\{x \mathbb{1}_{\left\{a=a_{j}\right\}}: x \in[0, \kappa]\right\}, \\
& V^{\theta_{k}}=\{0\} \text { for any } k \in I \backslash\{i, j\}, \\
& c_{i}\left(x \mathbb{1}_{\left\{a=a_{i}\right\}}, \theta_{i}\right)=\frac{5}{3 \kappa} x^{2}, \text { for any } x \in[0, \kappa], \\
& c_{j}\left(x \mathbb{1}_{\left\{a=a_{j}\right\}}, \theta_{j}\right)=\frac{5}{2 \kappa} x^{2}, \text { for any } x \in[0, \kappa], \text { and } \\
& c_{k}\left(0, \theta_{k}\right)=0 \text { for all } k \in I \backslash\{i, j\} .
\end{aligned}
$$

for some $\kappa>0$.

First, consider $G(\theta)$. If $a_{i}$ is chosen, $i$ 's optimal choice of valuation should be

$$
\underset{x \in[0, \kappa]}{\arg \max }\left\{-\frac{5}{3 \kappa} x^{2}+x\right\}=\frac{3}{10} \kappa .
$$

If $a_{j}$ is chosen, $j$ 's optimal choice of valuation should be

$$
\underset{x \in[0, \kappa]}{\arg \max }\left\{-\frac{5}{2} x^{2}+x\right\}=\frac{1}{5} \kappa .
$$

\footnotetext{
${ }^{23}$ The parameters are equivalent to the example in Section 3 if $\kappa=10$.
} 
The social welfare achieved by $\left(\frac{3}{10} \kappa \mathbb{1}_{\left\{a=a_{i}\right\}}, 0\right)$ is $-\frac{3}{20} \kappa+\frac{3}{10} \kappa=\frac{3}{20} \kappa$ and the social welfare achieved by $\left(0, \frac{1}{5} \kappa \mathbb{1}_{\left\{a=a_{i}\right\}}\right)$ is $-\frac{1}{10} \kappa+\frac{1}{5} \kappa=\frac{1}{10} \kappa$. Thus, $\left\{\left(a_{i}, \tau\left(\frac{3}{10} \kappa \mathbb{1}_{\left\{a=a_{i}\right\}}, 0\right),\left(\frac{3}{10} \kappa \mathbb{1}_{\left\{a=a_{i}\right\}}, 0\right)\right)\right\}=$ $G(\theta)$ should hold.

Then consider any mechanism $m$ that implements $(\alpha, \tau)$ in the mechanism stage. We show that $\left(a_{j}, \tau\left(0, \frac{1}{5} \kappa \mathbb{1}_{\left\{a=a_{j}\right\}}\right),\left(0, \frac{1}{5} \kappa \mathbb{1}_{\left\{a=a_{j}\right\}}\right)\right) \in S P E(\theta, m)$. First, it is clear that the valuation of $j$ is a best response to $i$ 's choice 0 . Next, given $\bar{v}_{j} \equiv \frac{1}{5} \kappa \mathbb{1}_{\left\{a=a_{j}\right\}}$,

$$
\underset{v_{i} \in V^{\theta_{i}}}{\arg \max }\left\{-c_{i}\left(v_{i}, \theta_{i}\right)+v_{i}\left(\alpha\left(v_{i}, \bar{v}_{j}\right)\right)+\bar{v}_{j}\left(\alpha\left(v_{i}, \bar{v}_{j}\right)\right)\right\}=0
$$

holds. This occurs because given $j$ 's valuation $\bar{v}_{j}=\frac{1}{5} \kappa \mathbb{1}_{\left\{a=a_{j}\right\}}$, the equation is maximized when $a_{j}$ is chosen and $i$ does not make any investments. Since $(\alpha, \tau)$ is efficient and strategyproof, $\tau_{i}\left(\cdot, \bar{v}_{j}\right)$ is written as a Groves function (Green and Laffont, 1977): $\tau_{i}\left(v_{i}, \bar{v}_{j}\right)=g\left(\bar{v}_{j}\right)-$ $\bar{v}_{j}\left(\alpha\left(v_{i}, \bar{v}_{j}\right)\right)$. Hence,

$$
\begin{aligned}
& \underset{v_{i} \in V^{\theta_{i}}}{\arg \max }\left\{-c_{i}\left(v_{i}, \tilde{\theta}_{i}\right)+v_{i}\left(\alpha\left(v_{i}, \bar{v}_{j}\right)\right)-\tau_{i}\left(v_{i}, \bar{v}_{j}\right)\right\} \\
= & \underset{v_{i} \in V^{\theta_{i}}}{\arg \max }\left\{-c_{i}\left(v_{i}, \tilde{\theta}_{i}\right)+v_{i}\left(\alpha\left(v_{i}, \bar{v}_{j}\right)\right)-g\left(\bar{v}_{j}\right)+\bar{v}_{j}\left(\alpha\left(v_{i}, \bar{v}_{j}\right)\right)\right\} \\
= & \underset{v_{i} \in V^{\theta_{i}}}{\arg \max }\left\{-c_{i}\left(v_{i}, \tilde{\theta}_{i}\right)+v_{i}\left(\alpha\left(v_{i}, \bar{v}_{j}\right)\right)+\bar{v}_{j}\left(\alpha\left(v_{i}, \bar{v}_{j}\right)\right)\right\} \\
= & 0
\end{aligned}
$$

should hold for any cost type $\tilde{\theta}_{i} \in \Theta_{i}$, which means that 0 is the best response for $i$, and hence, we have $\left(a_{j}, \tau\left(0, \frac{1}{5} \kappa \mathbb{1}_{\left\{a=a_{j}\right\}}\right),\left(0, \frac{1}{5} \kappa \mathbb{1}_{\left\{a=a_{j}\right\}}\right)\right) \in S P E(\theta, m)$. However, this contradicts $G(\theta)=\operatorname{SPE}(\theta, m)$.

\section{A.4 Proof of Proposition 3}

For any efficient allocation rule $\alpha$, consider the following transfer rule $\tau$ :

$$
\tau_{i}(u)=u_{i}(\alpha(u))-\frac{1}{n} \sum_{i \in I} u_{i}(\alpha(u)) .
$$


It is clear that $\tau$ is budget-balanced. Then, we show that $(\alpha, \tau)$ is commitment-proof. For any $i \in I, u \in U, \tilde{u}_{i} \in U_{i}$,

$$
\begin{aligned}
& {\left[\tilde{u}_{i}\left(\alpha\left(\tilde{u}_{i}, u_{-i}\right)\right)-\tau_{i}\left(\tilde{u}_{i}, u_{-i}\right)\right]-\left[u_{i}(\alpha(u))-\tau_{i}(u)\right]-\max \left\{0, \max _{a \in A}\left\{\tilde{u}_{i}(a)-u_{i}(a)\right\}\right\} } \\
= & \frac{1}{n}\left\{\tilde{u}_{i}\left(\alpha\left(\tilde{u}_{i}, u_{-i}\right)\right)+\sum_{j \in I \backslash\{i\}} u_{j}\left(\alpha\left(\tilde{u}_{i}, u_{-i}\right)\right)\right\}-\frac{1}{n} \sum_{i \in I} u_{i}(\alpha(u))-\max _{a \in A}\left\{0, \max _{a \in A}\left\{\tilde{u}_{i}(a)-u_{i}(a)\right\}\right\} \\
= & \frac{1}{n}\left\{\tilde{u}_{i}\left(\alpha\left(\tilde{u}_{i}, u_{-i}\right)\right)-u_{i}\left(\alpha\left(\tilde{u}_{i}, u_{-i}\right)\right)+\sum_{i \in I} u_{i}\left(\alpha\left(\tilde{u}_{i}, u_{-i}\right)\right)-\sum_{i \in I} u_{i}(\alpha(u))\right\} \\
& -\max \left\{0, \max _{a \in A}\left\{\tilde{u}_{i}(a)-u_{i}(a)\right\}\right\} \\
\leq & \frac{1}{n}\left\{\tilde{u}_{i}\left(\alpha\left(\tilde{u}_{i}, u_{-i}\right)\right)-u_{i}\left(\alpha\left(\tilde{u}_{i}, u_{-i}\right)\right)\right\}-\max \left\{0, \max _{a \in A}\left\{\tilde{u}_{i}(a)-u_{i}(a)\right\}\right\} \\
\leq & \frac{1}{n} \max \left\{0, \max _{a \in A}\left\{\tilde{u}_{i}(a)-u_{i}(a)\right\}\right\}-\max \left\{0, \max _{a \in A}\left\{\tilde{u}_{i}(a)-u_{i}(a)\right\}\right\} \\
= & -\frac{n-1}{n} \max \left\{0, \max _{a \in A}\left\{\tilde{u}_{i}(a)-u_{i}(a)\right\}\right\} \\
\leq & 0,
\end{aligned}
$$

where the first inequality holds from the efficiency of $\alpha$. Thus, this $(\alpha, \tau)$ is commitmentproof and the proof is done.

\section{A.5 Proof of Proposition 4}

Take any discount factor $\delta \in(0,1)$ and fix it.

Since $(\alpha, \tau)$ is strategy-proof, it satisfies Bayesian incentive compatibility for any $(\theta, v, \sigma)$. As in the if part in Theorem 1 , since the equilibrium ex post investment choice of every agent is always socially efficient, it suffices to show that for any $\theta \in \Theta$, the PBE ex ante investments are characterized by $Z^{\theta}$.

Consider the investment game induced by $(\alpha, \tau)$ given $\delta$, and consider any agent $i \in I$ and her cost type $\theta_{i} \in \Theta_{i}$. Every agent's ex post investment strategy is fixed to the PBE strategy $\mu^{*}$, and let us take any arbitrary ex ante investment strategies $\sigma_{-i} \in \Sigma_{-i}$ of other agents. Take any $v_{i}^{0} \in Z^{\theta_{i}}$ and any $v_{i} \in V^{\theta_{i}} \backslash Z^{\theta_{i}}$. We can show that $v_{i}^{0}$ gives a strictly higher utility than $v_{i}$ for agent $i$ of cost type $\theta_{i}$. To see this, the ex ante utility from $\left(v_{i}, \mu_{i}^{*}\right)$ 
given $\sigma_{-i}$ is written as:

$$
\begin{aligned}
& \delta \sum_{\theta_{-i} \in \Theta_{-i}} p\left(\theta_{-i} \mid \theta_{i}\right)\left[\mu_{i}^{*}\left(v_{i}, \sigma_{-i}\left(\theta_{-i}\right), \alpha\left(u^{\theta_{i}, v_{i}}, u^{\theta_{-i}, \sigma_{-i}\left(\theta_{-i}\right)}\right), \theta_{i}\right)\left(\alpha\left(u^{\theta_{i}, v_{i}}, u^{\theta_{-i}, \sigma_{-i}\left(\theta_{-i}\right)}\right)\right)\right. \\
& \left.-\tau_{i}\left(u^{\theta_{i}, v_{i}}, u^{\theta_{-i}, \sigma_{-i}\left(\theta_{-i}\right)}\right)-c_{i}\left(\mu_{i}^{*}\left(v_{i}, \sigma_{-i}\left(\theta_{-i}\right), \alpha\left(u^{\theta_{i}, v_{i}}, u^{\theta_{-i}, \sigma_{-i}\left(\theta_{-i}\right)}\right), \theta_{i}\right), \theta_{i}\right)\right] \\
& -(1-\delta) c_{i}\left(v_{i}, \theta_{i}\right) \\
< & \delta \sum_{\theta_{-i} \in \Theta_{-i}} p\left(\theta_{-i} \mid \theta_{i}\right)\left[\mu_{i}^{*}\left(v_{i}, \sigma_{-i}\left(\theta_{-i}\right), \alpha\left(u^{\theta_{i}, v_{i}}, u^{\theta_{-i}, \sigma_{-i}\left(\theta_{-i}\right)}\right), \theta_{i}\right)\left(\alpha\left(u^{\theta_{i}, v_{i}}, u^{\theta_{-i}, \sigma_{-i}\left(\theta_{-i}\right)}\right)\right)\right. \\
& \left.-\tau_{i}\left(u^{\theta_{i}, v_{i}}, u^{\theta_{-i}, \sigma_{-i}\left(\theta_{-i}\right)}\right)-c_{i}\left(\mu_{i}^{*}\left(v_{i}, \sigma_{-i}\left(\theta_{-i}\right), \alpha\left(u^{\theta_{i}, v_{i}}, u^{\theta_{-i}, \sigma_{-i}\left(\theta_{-i}\right)}\right), \theta_{i}\right), \theta_{i}\right)\right] \\
= & \delta \sum_{\theta_{-i} \in \Theta_{-i}} p\left(\theta_{-i} \mid \theta_{i}\right)\left[u^{\theta_{i}, v_{i}}\left(\alpha\left(u^{\theta_{i}, v_{i}}, u^{\theta_{-i}, \sigma_{-i}\left(\theta_{-i}\right)}\right)\right)-\tau_{i}\left(u^{\theta_{i}, v_{i}}, u^{\theta_{-i}, \sigma_{-i}\left(\theta_{-i}\right)}\right)-c_{i}\left(v_{i}, \theta_{i}\right)\right] \\
\leq & \delta \sum_{\theta_{-i} \in \Theta_{-i}} p\left(\theta_{-i} \mid \theta_{i}\right)\left[u^{\theta_{i}, v_{i}}\left(\alpha\left(u^{\theta_{i}, v_{i}}, u^{\theta_{-i}, \sigma_{-i}\left(\theta_{-i}\right)}\right)\right)-\tau_{i}\left(u^{\theta_{i}, v_{i}}, u^{\theta_{-i}, \sigma_{-i}\left(\theta_{-i}\right)}\right)\right. \\
& \left.\left.-\max _{\leq} 0, \max _{a \in A}\left\{u^{\theta_{i}, v_{i}}(a)-u^{\theta_{i}, v_{i}^{0}}(a)\right\}\right\}\right] \\
& \delta \sum_{\theta_{-i} \in \Theta_{-i}} p\left(\theta_{-i} \mid \theta_{i}\right)\left[u^{\theta_{i}, v_{i}^{0}}\left(\alpha\left(u^{\theta_{i}, v_{i}^{0}}, u^{\theta_{-i}, \sigma_{-i}\left(\theta_{-i}\right)}\right)\right)-\tau_{i}\left(u^{\theta_{i}, v_{i}^{0}}, u^{\theta_{-i}, \sigma_{-i}\left(\theta_{-i}\right)}\right)\right] \\
= & \delta \sum_{\theta_{-i} \in \Theta_{-i}} p\left(\theta_{-i} \mid \theta_{i}\right)\left[\mu_{i}^{*}\left(v_{i}^{0}, \sigma_{-i}\left(\theta_{-i}\right), \alpha\left(u^{\theta_{i}, v_{i}^{0}}, u^{\theta_{-i}, \sigma_{-i}\left(\theta_{-i}\right)}\right), \theta_{i}\right)\left(\alpha\left(u^{\theta_{i}, v_{i}^{0}}, u^{\theta_{-i}, \sigma_{-i}\left(\theta_{-i}\right)}\right)\right)\right. \\
& \left.-\tau_{i}\left(u^{\theta_{i}, v_{i}^{0}}, u^{\theta_{-i}, \sigma_{-i}\left(\theta_{-i}\right)}\right)-c_{i}\left(\mu_{i}^{*}\left(v_{i}^{0}, \sigma_{-i}\left(\theta_{-i}\right), \alpha\left(u^{\theta_{i}, v_{i}^{0}}, u^{\theta_{-i}, \sigma_{-i}\left(\theta_{-i}\right)}\right), \theta_{i}\right), \theta_{i}\right)\right],
\end{aligned}
$$

in which the last equation (26)-(27) is the ex ante utility from $\left(v_{i}^{0}, \mu_{i}^{*}\right)$ given $\sigma_{-i}$. The inequality in (20) holds because $c_{i}\left(v_{i}, \theta_{i}\right)>0$ and $\delta<1$; the equality in (22) follows from the definition of $u^{\theta_{i}, v_{i}}$; the inequality in (23) follows from Lemma 1; the inequality in (25) follows from the fact that $(\alpha, \tau)$ is commitment-proof because it is strategy-proof; and the equality in (26) follows from the definition of $u^{\theta_{i}, v_{i}^{0}}$. Therefore, for any strategies $\sigma_{-i}$ of other agents, the PBE ex ante investment for $i$ of cost type $\theta_{i}$ is characterized by $Z^{\theta_{i}}$, which implies $F(\theta)=\operatorname{PBE}(\theta, \alpha, \tau, \delta)$ for any $\theta \in \Theta$, and the proof is done. 